\title{
Modeling Degradation in Solid Oxide Electrolysis Cells - Vol II
}

Manohar S. Sohal Sergey N. Rashkeev Michael V. Glazoff Anil V. Virkar

September 2011

The INL is a

U.S. Department of Energy National Laboratory

operated by

Battelle Energy Alliance

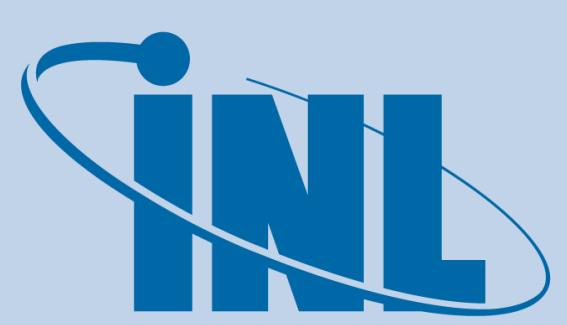

Idaho National Laboratory

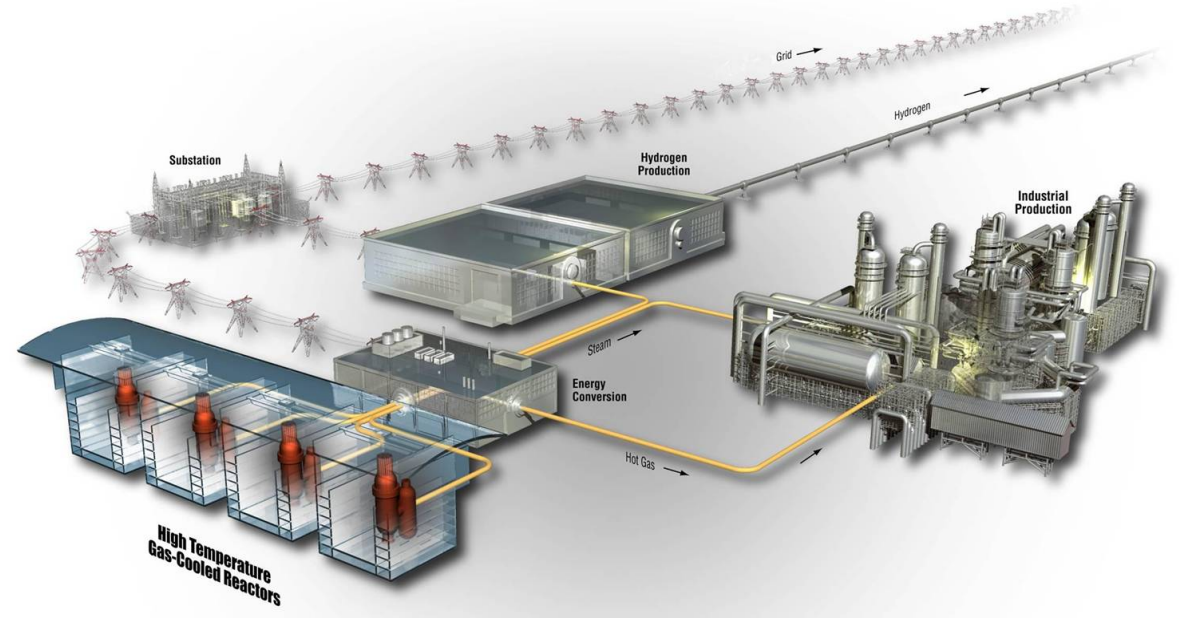




\section{DISCLAIMER}

This information was prepared as an account of work sponsored by an agency of the U.S. Government. Neither the U.S. Government nor any agency thereof, nor any of their employees, makes any warranty, expressed or implied, or assumes any legal liability or responsibility for the accuracy, completeness, or usefulness, of any information, apparatus, product, or process disclosed, or represents that its use would not infringe privately owned rights. References herein to any specific commercial product, process, or service by trade name, trade mark, manufacturer, or otherwise, does not necessarily constitute or imply its endorsement, recommendation, or favoring by the U.S. Government or any agency thereof. The views and opinions of authors expressed herein do not necessarily state or reflect those of the U.S. Government or any agency thereof. 


\section{Modeling Degradation in Solid Oxide Electrolysis Cells - Vol II}

Manohar S. Sohal Sergey N. Rashkeev Michael V. Glazoff

Anil V. Virkar (University of Utah)

September 2011

Idaho National Laboratory Idaho Falls, Idaho 83415

http://www.inl.gov

Prepared for the

U.S. Department of Energy Office of Nuclear Energy Under DOE Idaho Operations Office Contract DE-AC07-05ID14517 



\title{
Modeling Degradation in Solid Oxide Electrolysis Cells - Vol II
}

\author{
INL/EXT-11-23089
}

September 2011

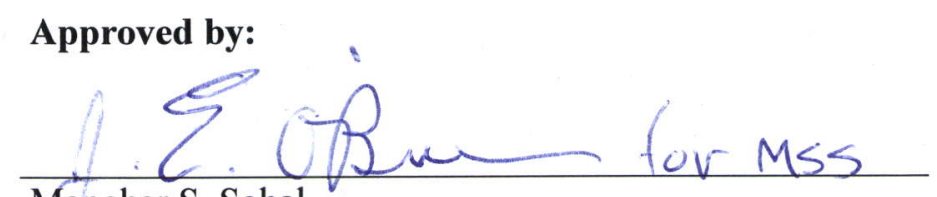

Manohar S. Sohal

Lead Author

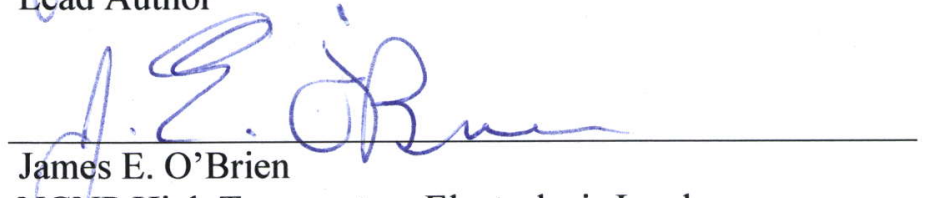

NGNP High Temperature Electrolysis Lead
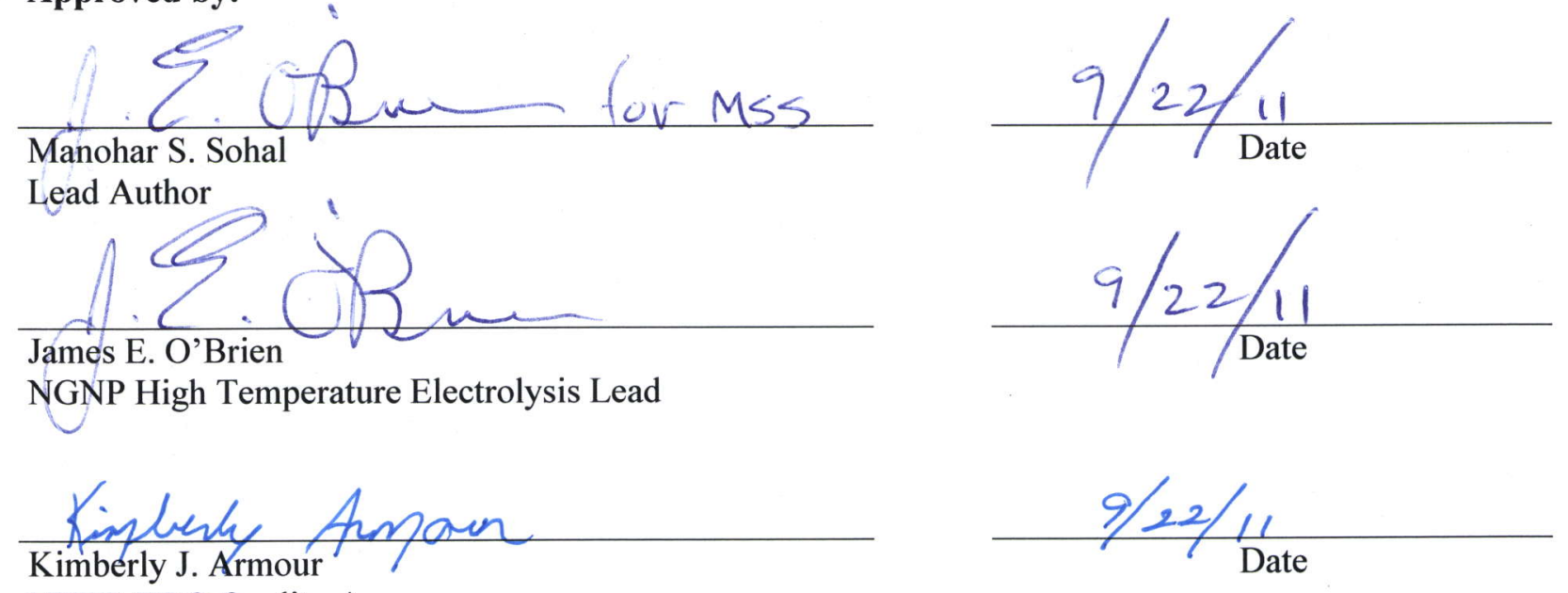

VHTR TDO Quality Assurance
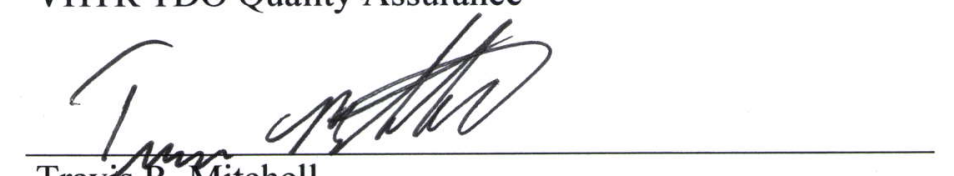

Travis R. Mitchell

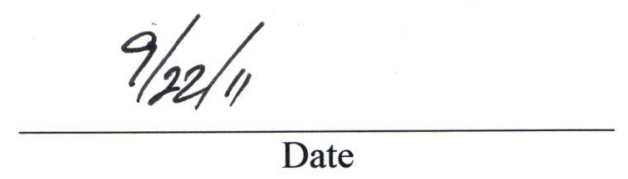

VHTR TDO Project Management 



\begin{abstract}
The Idaho National Laboratory (INL) has an ongoing project that supports the development of high-temperature electrolysis for efficient hydrogen production from steam using solid oxide electrolysis cells (SOECs). To accomplish this objective, technical and degradation issues associated with the SOECs must be addressed. This report covers various approaches being pursued to model degradation issues in SOECs.

Two separate degradation models have been developed. In one model, the equilibrium thermodynamics, non-equilibrium diffusion modeling, and first -principles, atomic-scale calculations were combined to understand the degradation mechanisms and to provide practical recommendations on how to inhibit them.

Another SOEC degradation model is based on the concept of local thermodynamic equilibrium in systems that are otherwise in global thermodynamic non-equilibrium. It is shown that electronic conduction through the electrolyte, however small, must be taken into account. Under certain conditions, high pressures can be generated electrochemically in the electrolyte just near the oxygen electrode/electrolyte interface, leading to oxygen electrode delamination. Development of high pressures may be avoided by introducing some electronic conductivity in the electrolyte.

Both models indicate that the addition of a small amount of a transition metal oxide or other oxides with cations exhibiting multiple valence states, such as ceria, to the electrolyte should increase its electronic conductivity and decrease the tendency for delamination of the oxygen electrode.
\end{abstract}




\section{ACKNOWLEDGEMENTS}

This report represents a compilation of the efforts of several researchers at Idaho National Laboratory and University of Utah. The editor acknowledges the original contribution of these researchers (listed as coauthors of this report). 


\section{CONTENTS}

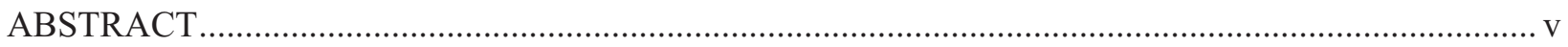

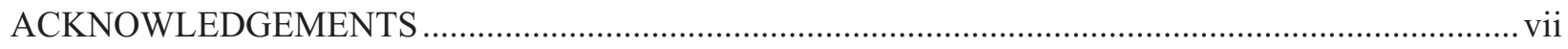

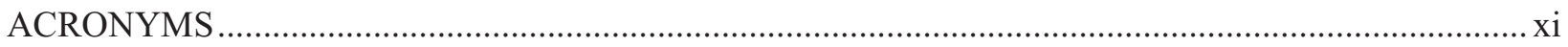

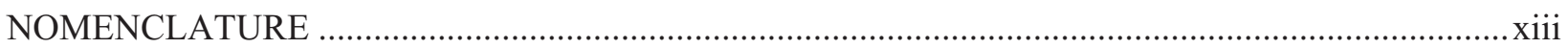

1. INTRODUCTION TO HIGH-TEMPERATURE ELECTROLYSIS WITH SOLID OXIDE

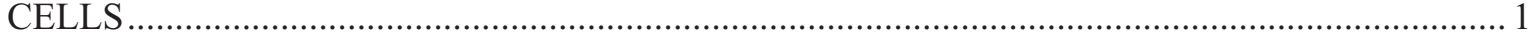

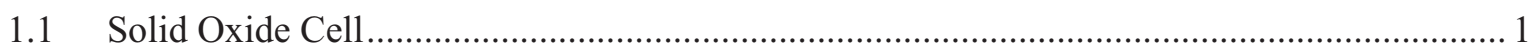

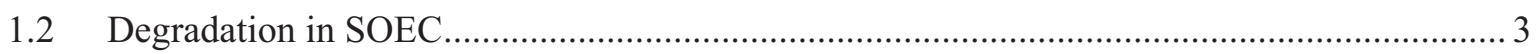

2. ATOMIC-SCALE MODELING OF OXYGEN ELECTRODE DELAMINATION IN

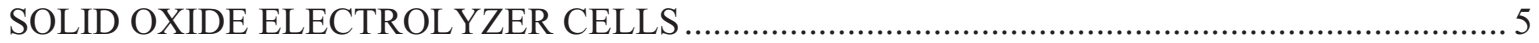

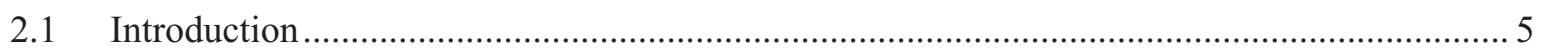

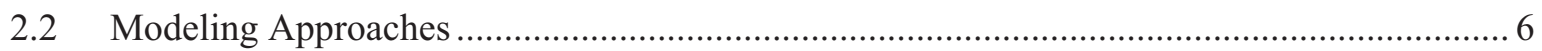

2.2.1 First-principles Calculations and Atomic-scale Modeling ....................................... 6

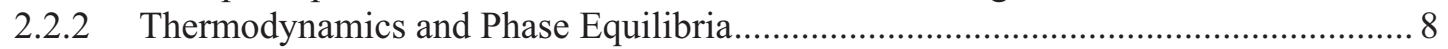

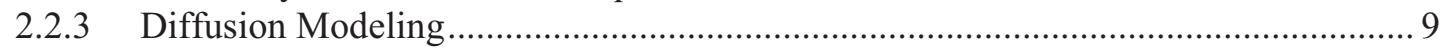

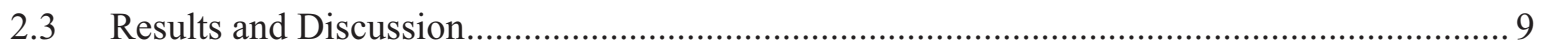

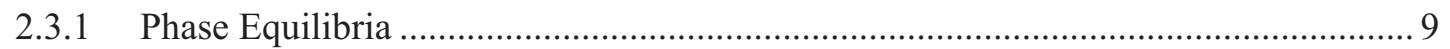

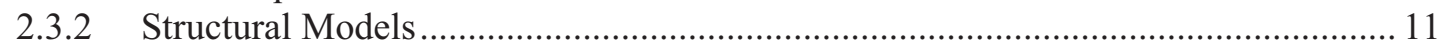

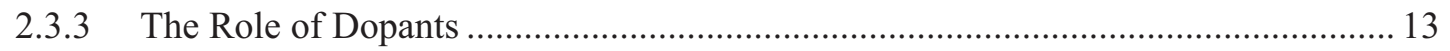

2.3.4 Molecular Oxygen Formation and Interfacial Pressure Development...................... 15

2.3.5 Improving operation of SOEC devices by interface modifications ......................... 18

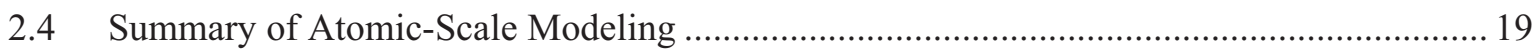

3. NON-EQUILIBRIUM THERMODYNAMICS MODELING OF DEGRADATION IN

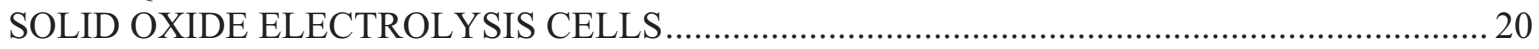

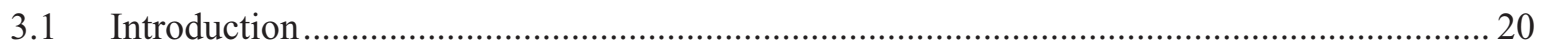

3.1.1 Global Non-equilibrium, Local Equilibrium......................................................... 20

3.2 Analysis of Currents through a Cell .................................................................................. 21

3.2.1 Ionic and Electronic Currents through the Cell and the Measured Current ............... 21

3.2.2 Delamination resulting from Development of High Oxygen Pressure in the Electrolyte near the Oxygen Electrode ................................................................ 23

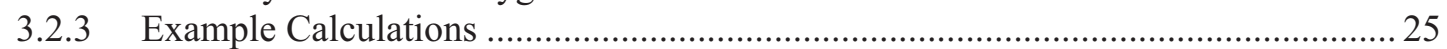

3.2.4 Development of Low Oxygen Pressure within the Electrolyte just near the Steam- $\mathrm{H}_{2}$ Electrode/Electrolyte Interface …....................................................... 26

3.3 Implication of Non-equilibrium Thermodynamic Modeling .......................................... 27

3.3.1 Implications concerning solid oxide fuel cells (SOFC) and solid oxide

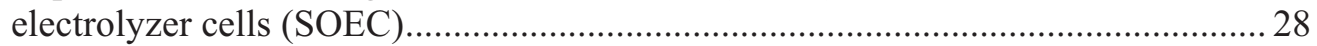

3.3.2 Prevention/Suppression of Oxygen Electrode Delamination.................................... 29

3.4 Summary of Non-equilibrium Thermodynamic Modeling ................................................. 30

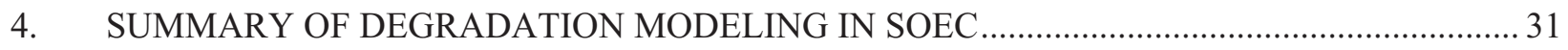




\section{FIGURES}

Figure 1. (a) SOEC; (b) solid oxide fuel cell (SOFC) operating in reverse compared to an SOEC............2

Figure 2. Ceramatec solid oxide cell/stack construction (scanning electron microscopy figure on the right is taken from Carter et al. 2008).

Figure 3. SEM micrographs from Figure 16 of Mawdsley et al. 2009 showing oxygen electrode delamination along the oxygen electrode/electrolyte interface.

Figure 4. Atomic-scale models for YSZ surface and LSM/YSZ interface: (a) a free (001) surface for the oxygen-saturated stabilized cubic $\mathrm{ZrO}_{2}$ structure; (b) a smooth $\mathrm{LaMnO}_{3} / \mathrm{ZrO}_{2}$ interface with (001) orientation. Oxygen atoms are shown in red, $\mathrm{Zr}$ in small light blue balls, $\mathrm{La}$ in big cyan balls, $\mathrm{Mn}$ in purple. Only three layers of $\mathrm{LaMnO}_{3}$ perovskite (nearest to the interface) are shown.

Figure 5. Free YSZ (001) surface, schematics of Y (a), Mn (b), and La (c) subsurface substitutional defects. In each case, two subsurface $\mathrm{Zr}$ atoms are substituted by dopant impurity atoms and one oxygen vacancy is added. Oxygen atoms are shown in red, $\mathrm{Zr}$ in small light blue balls, Y in yellow, La in big cyan balls, Mn in purple.

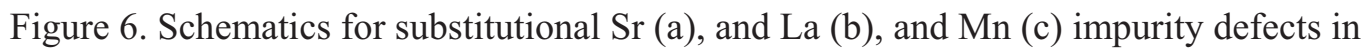
subsurface area of YSZ near the LSM/YSZ interface. A whole row of Zr atoms in the supercell was substituted by impurities. A defect-free interface is shown for comparison (d). Oxygen atoms are shown in red, $\mathrm{Zr}$ in small light blue balls, La in big cyan balls, $\mathrm{Mn}$ in purple, $\mathrm{Sr}$ in green. Only three layers of $\mathrm{LaMnO}_{3}$ perovskite (nearest to the interface) are shown. La-O and Sr-O bonds were omitted in figures (a) and (b) for better illustration.

Figure 7. (a) Schematics of the formation of peroxy O-O bonds (precursors of $\mathrm{O}_{2}$ molecules) at the (001) surface of cubic $\mathrm{ZrO}_{2}$ related to substitutional $\mathrm{Mn}$ impurities in the subsurface region. Oxygen atoms are shown in red, $\mathrm{Zr}$ - as small light blue balls, $\mathrm{Mn}$ - in purple; (b) Equilibrium pressure of $\mathrm{O}_{2}$ gas above a free $\mathrm{ZrO}_{2}$ surface at $T=800^{\circ} \mathrm{C}$ as a function of $\mathrm{O}_{2}$ formation energy corresponding to different subsurface substitutional defects. 16

Figure 8. A schematic of a solid oxide electrolyzer cell, when operated in the electrolyzer mode.

Figure 9. An equivalent circuit for the cell in a "true" steady state ........................................................22

Figure 10. An equivalent circuit for the cell in an "apparent" steady state.

\section{TABLES}

Table 1. Commonly used materials in SOFC/SOEC. ${ }^{3}$ 


\section{ACRONYMS}

ASR area-specific resistance

bcc body-centered cubic

CGO cerium-gadolinium oxide

DFT Density Functional Theory

EDS energy dispersive $\mathrm{x}$-ray spectroscopy

EFTEM Energy Filtered Transmission Electron Microscopy

fcc face-centered cubic

GDC gadolinia-doped ceria

GGA generalized gradient approximation

GUESS Gaussians Used for Embedded System Studies

INL Idaho National Laboratory

KMC Kinetic Monte Carlo

LSC lanthanum strontium cobalt oxide

LSCF lanthanum strontium cobaltite ferrite $\left(\mathrm{LaSr}(\mathrm{Co}, \mathrm{Fe}) \mathrm{O}_{3}\right)$

LSF strontium-doped lanthanum ferrite

LSM strontium-doped lanthanum manganite $\left(\mathrm{LaMnO}_{3}\right)$

MD molecular dynamics

NEB nudge elastic band

Ni-YSZ nickel-yttria stabilized zirconia

PAW projected augmented wave

PBE Perdew, Burke, and Ernzerhof

SDC samaria-doped ceria

SEM scanning electron microscopy

SOEC solid oxide electrolysis cell

SOFC solid oxide fuel cell

TEM transmission electron microscopy

TPB triple-phase boundary

VASP Vienna Atomic Simulation Program

YSZ yttria-stabilized zirconia 


\section{NOMENCLATURE}

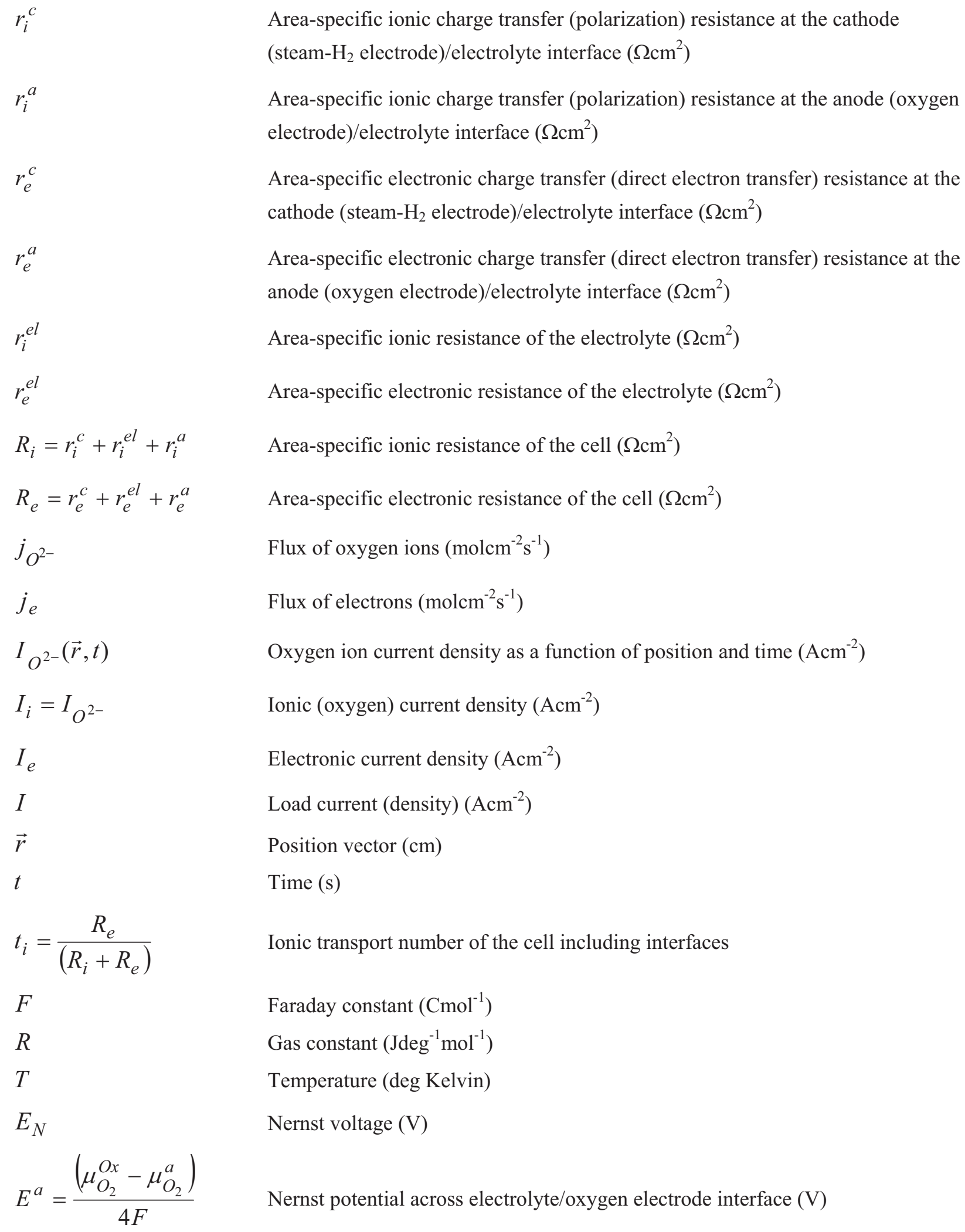$$
r_{i}^{c}
$$$$
r_{i}^{a}
$$$$
r_{e}^{c}
$$$$
r_{e}^{a}
$$$$
r_{i}^{e l}
$$$$
r_{e}^{e l}
$$$$
R_{i}=r_{i}^{c}+r_{i}^{e l}+r_{i}^{a}
$$$$
R_{e}=r_{e}^{c}+r_{e}^{e l}+r_{e}^{a}
$$$$
j_{O^{2-}}
$$$$
j_{e}
$$$$
I_{O^{2-}}(\vec{r}, t)
$$$$
I_{i}=I_{O^{2-}}
$$$$
I_{e}
$$$$
\text { I }
$$$$
\vec{r}
$$$$
t
$$$$
t_{i}=\frac{R_{e}}{\left(R_{i}+R_{e}\right)}
$$$$
F
$$$$
\text { R }
$$$$
T
$$$$
E_{N}
$$$$
E^{a}=\frac{\left(\mu_{O_{2}}^{O x}-\mu_{O_{2}}^{a}\right)}{4 F}
$$

Area-specific ionic charge transfer (polarization) resistance at the cathode (steam- $\mathrm{H}_{2}$ electrode)/electrolyte interface $\left(\Omega \mathrm{cm}^{2}\right)$

Area-specific ionic charge transfer (polarization) resistance at the anode (oxygen electrode)/electrolyte interface $\left(\Omega \mathrm{cm}^{2}\right)$

Area-specific electronic charge transfer (direct electron transfer) resistance at the cathode (steam- $\mathrm{H}_{2}$ electrode)/electrolyte interface $\left(\Omega \mathrm{cm}^{2}\right)$

Area-specific electronic charge transfer (direct electron transfer) resistance at the anode (oxygen electrode)/electrolyte interface $\left(\Omega \mathrm{cm}^{2}\right)$

Area-specific ionic resistance of the electrolyte $\left(\Omega \mathrm{cm}^{2}\right)$

Area-specific electronic resistance of the electrolyte $\left(\Omega \mathrm{cm}^{2}\right)$

Area-specific ionic resistance of the cell $\left(\Omega \mathrm{cm}^{2}\right)$

Area-specific electronic resistance of the cell $\left(\Omega \mathrm{cm}^{2}\right)$

Flux of oxygen ions $\left(\mathrm{molcm}^{-2} \mathrm{~s}^{-1}\right)$

Flux of electrons $\left(\mathrm{molcm}^{-2} \mathrm{~s}^{-1}\right)$

Oxygen ion current density as a function of position and time $\left(\mathrm{Acm}^{-2}\right)$

Ionic (oxygen) current density $\left(\mathrm{Acm}^{-2}\right)$

Electronic current density $\left(\mathrm{Acm}^{-2}\right)$

Load current (density) $\left(\mathrm{Acm}^{-2}\right)$

Position vector $(\mathrm{cm})$

Time (s)

Ionic transport number of the cell including interfaces

Faraday constant $\left(\mathrm{Cmol}^{-1}\right)$

Gas constant $\left(\mathrm{Jdeg}^{-1} \mathrm{~mol}^{-1}\right)$

Temperature (deg Kelvin)

Nernst voltage (V)

Nernst potential across electrolyte/oxygen electrode interface (V) 


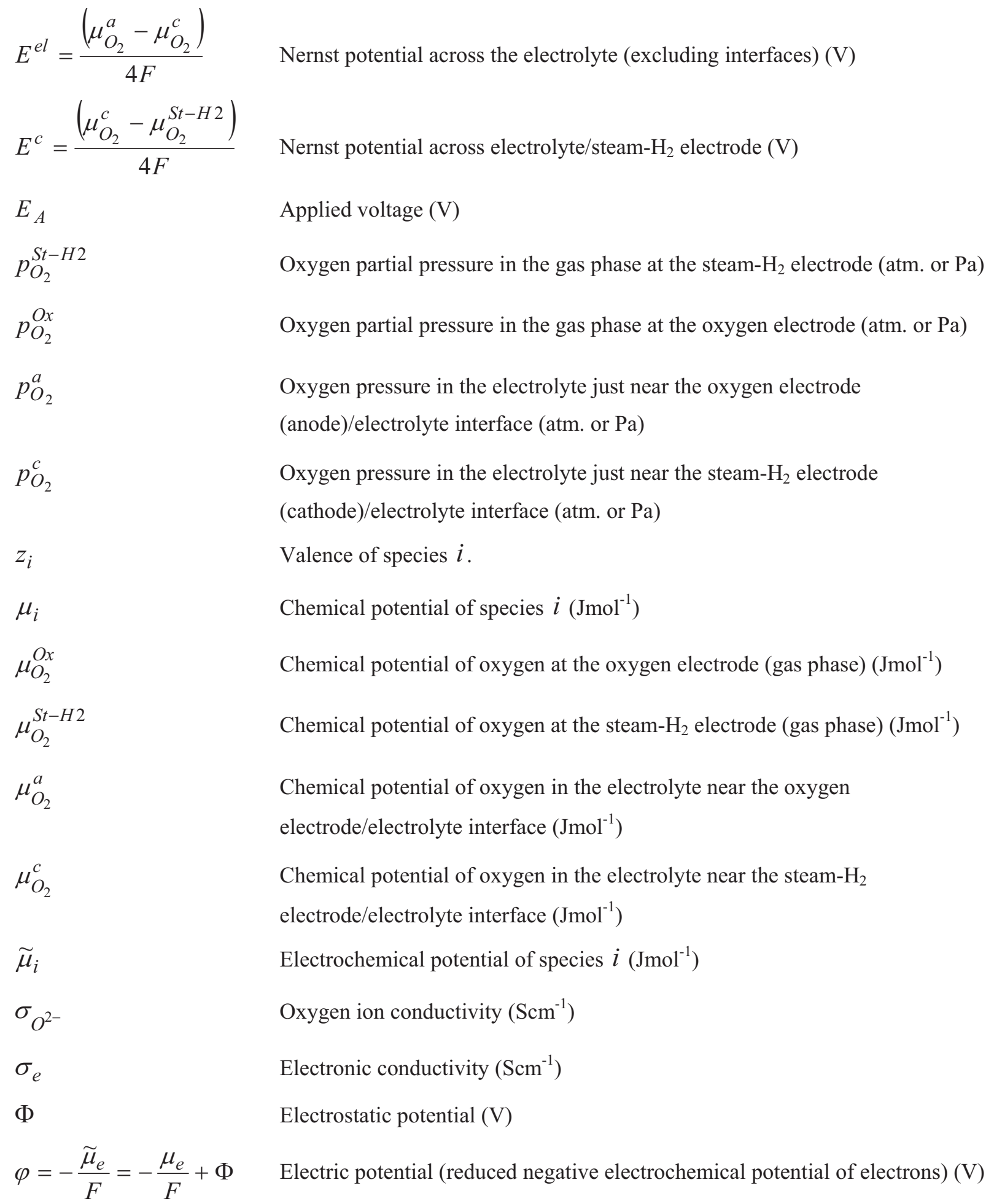




\section{Modeling Degradation in Solid Oxide Electrolysis Cells - Vol II}

\section{INTRODUCTION TO HIGH-TEMPERATURE ELECTROLYSIS WITH SOLID OXIDE CELLS}

The Idaho National Laboratory (INL) is performing high-temperature electrolysis research to generate hydrogen using solid oxide electrolysis cells (SOECs). This concept produces hydrogen using the heat and electricity generated by a high-temperature nuclear reactor. The combination of a high-efficiency power cycle and the direct utilization of nuclear process heat can result in overall thermal-to-hydrogen conversion efficiencies of $50 \%$ or higher. The objective of the INL project is to address a broad range of SOEC issues, including identification and evaluation of cell performance degradation parameters and processes, cell degradation modeling, integrity, and reliability of the SOEC stacks.

A mixture of steam and hydrogen is fed to the electrolytic cells where the oxygen migrates through the solid-state electrolyte as $\mathrm{O}^{=}$ions because of the imposed voltage. The cell voltage is significantly lower than that required in conventional electrolyzers because of the high operating temperature. In addition, electrolytic reaction kinetics is much faster than in room-temperature electrolyzers, thus minimizing polarization losses. The mixture exiting the cells, about $25 \mathrm{vol} \%$ steam and $75 \mathrm{vol} \%$ hydrogen, enters a separator where the two components are separated either by condensation of the steam or diffusion of the hydrogen. A fraction of the produced hydrogen is recycled and mixed with inlet steam to achieve the required inlet mixture composition, maintaining reducing conditions on the oxygen electrode. It is then reheated and returned to the solid oxide cells. The remaining steam can be removed from the hydrogen product stream through condensation. Like the hydrogen stream, the oxygen stream exiting the cells has a temperature of about $830^{\circ} \mathrm{C}$, which can be effectively used as needed.

\subsection{Solid Oxide Cell}

A solid oxide cell consists of three main components: an electrolyte and two electrodes (Figure 1 and Figure 2). The electrolyte is a ceramic membrane that can conduct ions and is sandwiched between two porous electrodes that can conduct electrons: the steam/hydrogen electrode (or anode in fuel-cell mode) and the air/oxygen electrode (or cathode in the fuel-cell mode). In the fuel-cell mode, oxygen molecules dissociate at the oxygen electrode and combine with electrons coming from an external electric power source to form oxygen ions. The oxygen ions conduct through the electrolyte and migrate towards the hydrogen electrode. The fuel (hydrogen or natural gas) is fed to the anode and reacts with the oxygen ions to form water and $\mathrm{CO}_{2}$. If the fuel cell is operated in the electrolysis mode, the names and function of the electrode get reversed, as shown in Figure 1. 


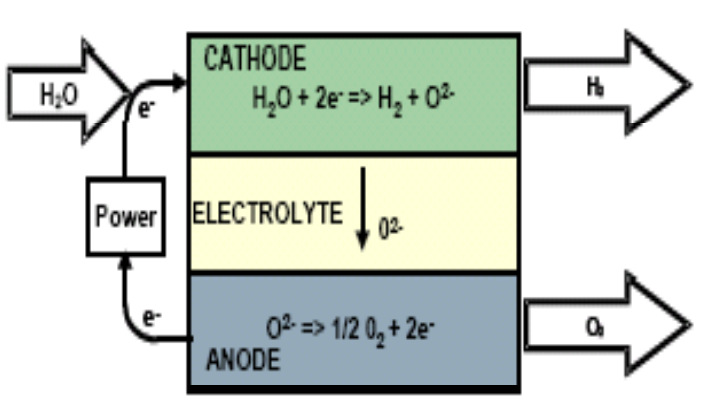

(a)

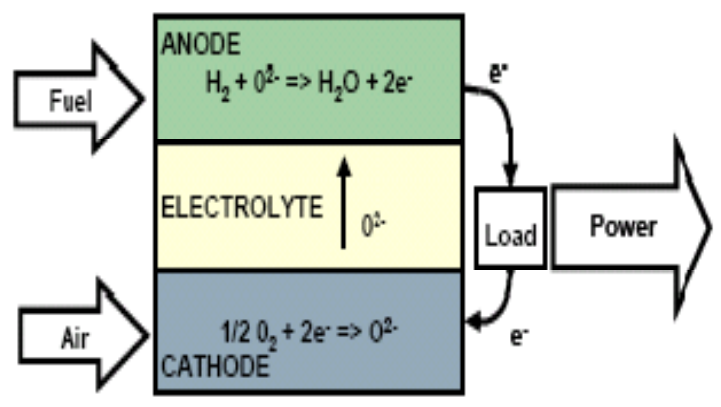

(b)

Figure 1. (a) SOEC; (b) solid oxide fuel cell (SOFC) operating in reverse compared to an SOEC. ${ }^{1}$
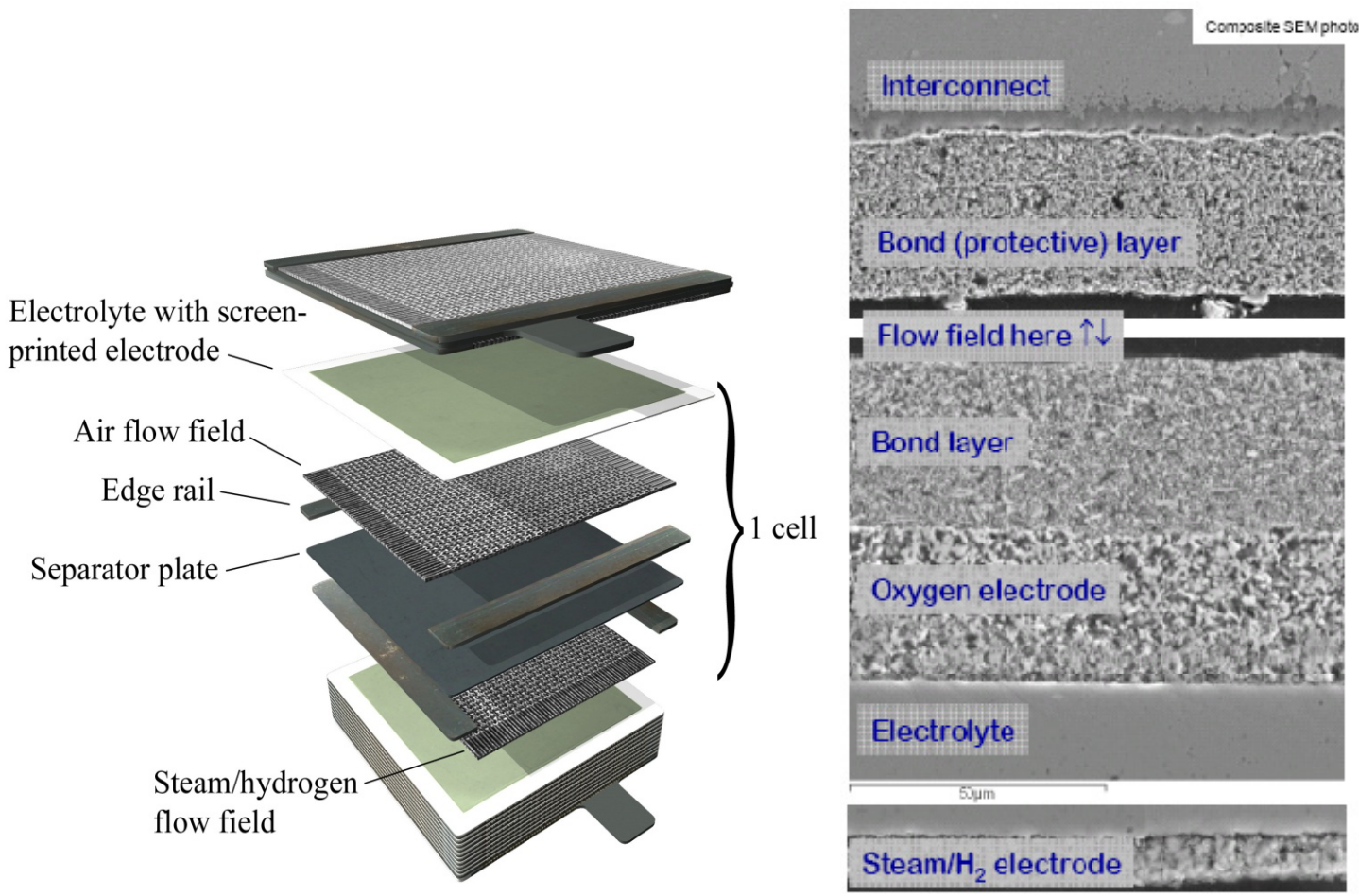

Figure 2. Ceramatec solid oxide cell/stack construction (scanning electron microscopy figure on the right is taken from Carter et al. 2008). ${ }^{2}$

The most common materials currently used for the solid oxide cells are listed in Table $1 .^{3}$ The electrolyte is a dense gas-tight ceramic layer, usually made from yttria-stabilized zirconia (YSZ) with yttria content of $8 \mathrm{~mol} \%$ to fully stabilize the electrolyte composition. The performance of the electrolyte depends on how well it can conduct oxide ions $\left(\mathrm{O}^{=}\right)$. The thinner the electrolyte, the higher its ion conductivity and the lower the cell's ohmic resistance. In an electrolyte supported cell, the electrolyte thickness is large $(150-250 \mu \mathrm{m})$, which leads to relatively high ionic resistance. Therefore, if the mechanical strength can be provided by the steam $/ \mathrm{H}_{2}$ electrode, the electrolyte thickness can be reduced by a factor of 10 or so. 
Table 1. Commonly used materials in SOFC/SOEC. ${ }^{3}$

\begin{tabular}{|l|l|l|}
\hline \multicolumn{1}{|c|}{ Component } & \multicolumn{1}{|c|}{ Material } & \multicolumn{1}{|c|}{ Acronym } \\
\hline Steam/hydrogen electrode & $\mathrm{Ni}^{-} \mathrm{Y}_{\mathrm{x}} \mathrm{Zr}_{1-\mathrm{x}} \mathrm{O}_{2-\mathrm{x} / 2}$ (nickel-YSZ) & Ni-YSZ \\
\hline Electrolyte & $\mathrm{Y}_{\mathrm{x}} \mathrm{Zr}_{1-\mathrm{x}} \mathrm{O}_{2-\mathrm{x} / 2}(\mathrm{YSZ})$ & $\mathrm{YSZ}$ \\
\hline Air/oxygen electrode & $\mathrm{Sr}_{\mathrm{x}} \mathrm{La}_{1-\mathrm{x}} \mathrm{MnO}_{3-\delta}+\mathrm{Y}_{\mathrm{x}} \mathrm{Zr}_{1-\mathrm{x}} \mathrm{O}_{2-\mathrm{x} / 2}$ (doped lanthanum manganite) & $\mathrm{LSM}$-YSZ \\
\hline Interconnect & Chromium-based alloys/ceramics or stainless steel & $\mathrm{SS}$ \\
\hline
\end{tabular}

The most common anode material for SOFC is a porous cermet (ceramic-metal) made from Ni and YSZ. Electronically conductive, gas-tight interconnect plates connect the individual cells to form a stack. The ionic conductivity of ceramics is highly dependent on the ceramic temperature. Thus, high operating temperatures are required to obtain sufficient overall conductivity in the solid oxide cell. YSZ exhibits acceptable conductivity in the $700-1,100^{\circ} \mathrm{C}$ temperature range, but if thermal cycling occurs, such as at start-up, the high operating temperature results in large thermal stresses in the cell components. Stresses can also be caused by large thermal gradients generated by the uneven distribution of electrochemical reaction sites. Finally, fabrication of the cell components also requires high temperatures that can cause detrimental residual stresses within the cell components.

In the fuel-cell mode, the oxygen electrode is fed with air while the hydrogen electrode is fed with hydrogen or natural gas. Thus, the properties of the oxygen electrode should be such that it provides a component for oxygen gas to be easily reduced and similarly, the function of the hydrogen electrode is to oxidize the fuel gas. At the oxygen electrode, where electrons are supplied via the external electrical power, oxygen molecules are reduced to oxygen ions. The oxygen ions are conducted through the electrolyte to the hydrogen electrode. At the hydrogen electrode, oxygen ions oxidize the fuel gas, which forms water and carbon dioxide, while the resulting free electrons are transported via the external circuit back to the oxygen electrode. The solid oxide electrolyte separates the reduction and oxidation reactions. Thus, in the electrolysis mode, the electrical energy is used to split hydrogen from steam. The electrolysis process is the reverse of the fuel-cell process.

\subsection{Degradation in SOEC}

Considerable work has been reported on the use of SOEC for electrolysis of $\mathrm{H}_{2} \mathrm{O}$ for hydrogen generation. The viability of SOEC for hydrogen generation as a practical system, however, has not been demonstrated in long-term testing since SOEC cells do degrade over time, and it is known that the degradation rate is typically greater than that in SOFCs, which often are identical (or similar) cells but operated in the power-generation mode. Some of the likely reasons of cell degradation include small initial compositional inhomogeneities resulting in large changes in properties, the formation of local hot spots leading to local changes in microstructures and material properties, electrode delamination caused by thermal cycling/rapid heating, reaction between electrode and electrolyte forming a high resistance layer, fuel and/or oxidant maldistribution, non-uniform oxidation of the interconnect, degradation of the seals, etc. An example of failure in SOECs is the occurrence of delamination of the oxygen electrode. As an example of the morphology of oxygen electrode delamination in SOEC, Figure 3 shows a scanning electron microscope (SEM) micrograph of a delaminated oxygen electrode. Background information on solid oxide cell degradation, polarization losses, and earlier efforts on degradation modeling in an SOEC are described in previous reports on the subject. ${ }^{4,5}$ Some of the material from previous reports on degradation $^{4,5}$ is being reproduced in this report for completeness. 

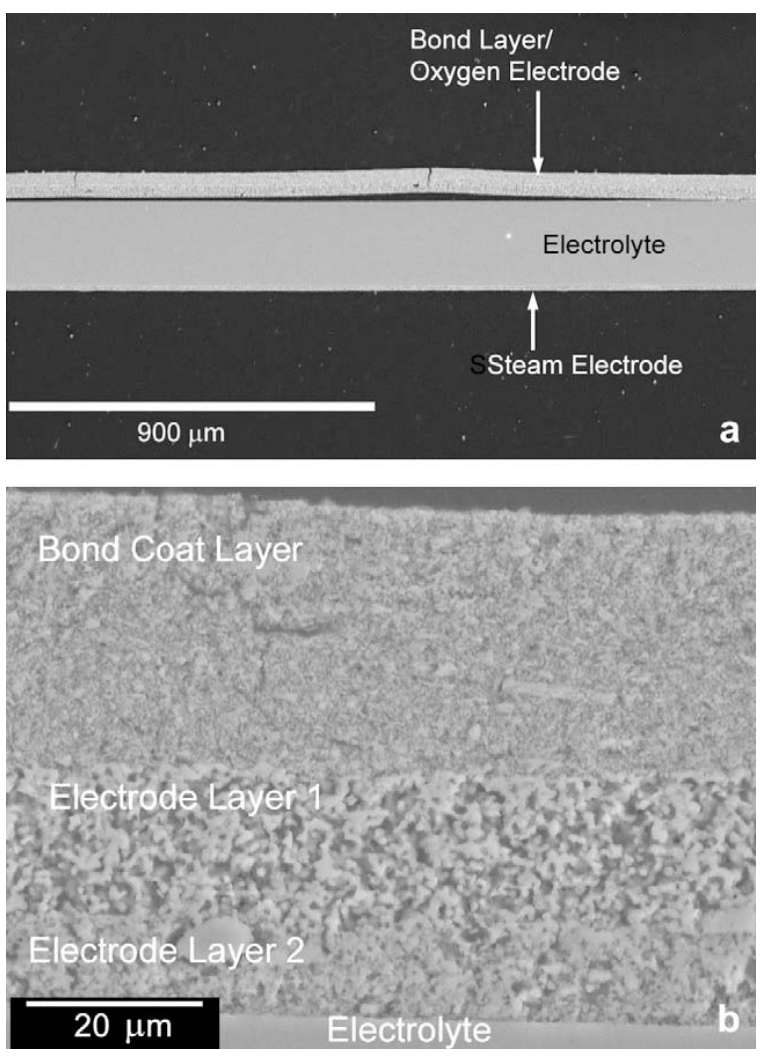

Figure 3. SEM micrographs from Figure 16 of Mawdsley et al. 2009 showing oxygen electrode delamination along the oxygen electrode/electrolyte interface. 


\title{
2. ATOMIC-SCALE MODELING OF OXYGEN ELECTRODE DELAMINATION IN SOLID OXIDE ELECTROLYZER CELLS
}

\author{
(Sergey N. Rashkeev and Michael V. Glazoff, INL)
}

\subsection{Introduction}

Solid-oxide devices operating at high-temperatures - SOFCs that produce electricity and SOECs that produce oxygen and hydrogen - place stringent requirements on materials used for cell construction. In fact, appropriate materials for cell components are very scarce. ${ }^{6}$ The most important consideration is matching the thermal expansion coefficients of electrode materials with that of the electrolyte to guarantee the high quality of the interfaces and to prevent cracking and/or delamination of the device components either during high-temperature operation or heating/cooling cycles. Therefore, two of the key technical challenges are the development of suitable materials and development of the fabrication technique.

Considerable work has been reported on the use of SOECs for hydrogen generation in the last few decades. . $^{7,9,10,11,12,13,14,15,16,17,18,19,20,21}$ To achieve robust performance targets (current density, tolerable degradation rate, and desired lifetime - about 20,000 hours), the existing SOEC technologies need to be improved. ${ }^{19}$ The Integrated Laboratory Scale test performed at the INL facility showed that a significant degradation of the SOEC stacks occurs over the first 1,000 hours, which is greater than degradation in a typical SOFC - a solid oxide device with a design identical or similar to that of a SOEC but operating in the power-generation mode. Therefore, the most important issue is to understand the degradation mechanisms of the SOEC devices and to find a way to significantly slow down the physical and chemical processes that cause such degradation.

Post test examination of SOECs showed that the hydrogen electrode appeared to be in good condition while the oxygen electrode delaminated from the electrolyte. ${ }^{1,16,19,21,22}$ One possible reason for delamination (which is considered one of the dominating SOEC degradation mechanisms) could be an excessive pressure buildup near the electrode-electrolyte interface. ${ }^{22}$ Indeed, high oxygen flux at the electrode-electrolyte interface may lead to a mismatch between the greater ability of zirconia to release oxygen and the lesser ability of the electrode material to release oxygen ions. If this is the case, then any defect at the solid-solid interface between the two materials could serve as a focal point where oxygen gas could be released after two electrons are transferred from each oxygen ion to the electrode. Under continuous operation, more and more oxygen would be accumulated around this defect, and pressure would build up. Eventually such a defect would grow into a crack, cleaving the perovskite--zirconia interface(s). When the oxygen electrode material in the cells is a mixture of zirconia and LSM perovskite grains, delimitation may also occur within the electrode but, in any case, close to the electrolyte interface. $^{21}$

Other degradation mechanisms may also contribute significantly, such as (i) transport of Crcontaining species from steel interconnects into the oxygen electrode and bond layers, ${ }^{23}$ (ii) cation segregation and phase separation processes in different SOEC components and their interfaces; ${ }^{24}$ (iii) the appearance of different contaminants (Ni, Cr, Si, etc.) on reaction sites; and (iv) the loss of electronic/ionic conductivity of electrolyte. Recent high-resolution SEM and transmission electron microscope (TEM) studies of the changes in the oxygen electrode/electrolyte interface microstructure for anode-supported technological SOFCs (subjected to long-term, $\sim 1500$-h testing at $750^{\circ} \mathrm{C}$ under high electrical loading) indicated that the interface degradation was caused by the size reduction of the individual LSM/YSZ electrolyte contact areas that were initially about $100-200 \mathrm{~nm}$ in diameter. ${ }^{24}$ Also, the interface exhibited an intensive interdiffusion process between the electrode and electrolyte- $\mathrm{Zr}$ atoms diffused into the LSM perovskite while La and Mn atoms diffused into the YSZ electrolyte - and some highly undesirable phases (e.g., zirconates) were formed. 
This research is aimed at gaining fundamental understanding of different factors affecting oxygen delamination processes in SOEC devices. On the basis of this understanding, we propose different possible improvements to the existing SOEC technology that would help in achieving the desirable performance targets for these devices as well as predict and rationally design other high-lifetime SOEC devices based on different materials. Heuristic methods are insufficient for fundamental studies of degradation processes in SOEC systems. These processes initiate and develop at different length scales (including atomic-, nano-, and micro-scales), and one needs to use a suitable combination of different experimental and computational approaches to address such a multi-scale, interdisciplinary problem.

SOEC materials (electrodes, electrolyte, steel interconnects, etc.) have to function in a very aggressive environment: at temperatures up to $850^{\circ} \mathrm{C}$, in a corrosive oxygen, hydrogen, and water vapor atmosphere in the presence of electric fields. It is critical to ensure that materials used for the SOEC devices (in this case, porous LSM) anode, nickel-zirconia cermet cathode, YSZ electrolyte, and ferritic steel interconnects) as well as their surfaces and interfaces do not undergo phase transformations or diffusion-related changes of chemical composition resulting in their degradation and loss of functionality.

The problem of material degradation in SOEC systems cannot be resolved successfully without a fundamental understanding of atomic and nano-scale defects, phase equilibria, complex diffusion-induced processes, and electrochemical phenomena. One of the goals was to understand, and consequently control, a number of degradation issues associated with phase decomposition and diffusion-induced phase transformations in SOEC. A combination of first-principles, density functional theory (DFT)-based calculations, and thermodynamic and diffusion modeling was used to understand the processes that contribute to the oxygen delamination in the electrolyte and at the oxygen electrode-electrolyte interface. A typical SOEC device that consists of YSZ electrolyte and LSM oxygen electrode was considered. In particular, we investigated different bulk, surface, and interface defects in these devices. It was found that delamination processes are significantly accelerated by high-temperature interdiffusion of different atoms across the LSM/YSZ interface (La, Sr, and Mn from LSM to YSZ, and Zr from YSZ to LSM). At the same time, different dopant atoms play different roles in YSZ; while La and Sr atoms with large atomic radii affect structural stability of the electrolyte and interface, Mn does not significantly reconfigure the electrolyte network but may increase electronic conductivity in the LSM/YSZ interfacial layer, which results in oxygen-gas formation and accumulation in closed defects (voids, pores, cracks, etc.). This gas will initiate a significant pressure buildup in the LSM/YSZ interfacial region, which will initiate and/or cause the oxygen electrode to delaminate. Simple models for estimating these effects are proposed. Identifying modeling based engineering solutions will help improve operation of the SOEC devices up to the point when they become economically feasible.

For that goal, we applied different methods of modeling: first-principles calculations and atomic-scale simulations, thermodynamics, and diffusion modeling. In particular, two novel advanced modeling tools were used: Thermo-Calc and Dictra. A brief description of all of the modeling approaches used and their role in understanding the SOEC materials and devices is described below.

\subsection{Modeling Approaches}

\subsubsection{First-principles Calculations and Atomic-scale Modeling}

Degradation processes in materials systems typically start with individual atomic-scale defects that are formed, interact with each other, and form different defects, instabilities, and imperfections at larger length scale, etc. Therefore, any serious study of degradation processes in materials and devices should start with atomic-scale investigations of point (localized in all three dimensions) defects. Calculations of defects and defect processes in materials have to account accurately for several physical phenomena such as trapping of the electronic charge, mechanisms of defect formation, defect-induced relaxation, photo-induced excitations, and formation of transient species. 
Three models are usually used in calculations. In the periodic model, a fragment of a material (supercell) is selected and translated periodically in three dimensions. In a molecular cluster model, a finite fragment of material (cluster) is used to model the defect and its nearest environment. Both schemes have their pros and cons. The third (embedded cluster) model attempts to combine the advantages of both of them. In this case, a defect with its nearest environment is considered using a high-level theory (usually $a b$ initio methods). At the same time, the effect of the remaining part of the system is taken into account using a less-accurate method, e.g., classical interatomic potential. One such scheme has been pioneered at University College of London and is now implemented is a computer code GUESS (Gaussians Used for Embedded System Studies). ${ }^{25}$

Using first-principles, self-consistent, density-functional-based electronic structure calculations, the formation energies and migration barriers were calculated for several different defects in solid solutions corresponding to the materials and interfaces used in SOECs. In particular, the role of different point defects (vacancies, single impurities, interstitials, etc.) in degradation of bulk materials and interfaces was investigated. Future plans are to investigate the role of extended defects (voids, dislocation cores, etc.) in the processes of transport and trapping of different atoms at interfaces (e.g., at the anode-electrolyte interface). Some calculations were performed for large clusters using the Gaussian 09 codes. ${ }^{26}$ Most of the calculations were performed for large periodic supercells (using plane waves and pseudopotentials implemented in the Vienna Atomic Simulation Program [VASP] codes). ${ }^{27,28,29}$ The most recent version of the VASP codes (VASP.5.2) has a significant number of different useful options such as hybrid density functionals and an approximation implementation method for excited electronic states, which provide an opportunity to perform very accurate and reliable calculations for different defects. We plan to use the values calculated from first-principles for parameterization the Kinetic Monte Carlo (KMC) codes for calculating different transport coefficients. Some molecular dynamics (MD) modeling using the LAMMPS codes developed at Sandia National Laboratory will also be used if necessary.

The problem of material degradation in an SOEC device cannot be resolved successfully without a fundamental understanding of atomic- and nano-scale defects, phase equilibria, complex diffusion-induced processes, and electrochemical phenomena. The main goal is to understand atomic-scale mechanisms of oxygen electrode delamination, i.e., separation of the LSM oxygen electrode from the YSZ electrolyte. For this purpose, first-principles calculations were performed for different impurity-related atomic-scale defects in LSM, YSZ, and at the LSM/YSZ interface. Different possibilities to inhibit delamination were considered by modifying the LSM/YSZ interface. A number of degradation issues associated with phase decomposition and diffusion-induced phase transformations in SOECs were also discussed using the analysis of existing data on heterogeneous phase equilibria in these systems.

Many degradation processes in materials start with individual atomic-scale defects that form, interact with each other, transform into another types of defects, and cause instabilities and imperfections at larger length scales. Therefore, any comprehensive study of degradation processes in materials and devices should start with atomic-scale investigations of point defects localized in all three dimensions. Modeling of defect behavior and defect-mediated processes in materials can explain several physical phenomena such as trapping of electronic charge, mechanisms of defect forming, defect-induced relaxation, and formation of transient species.

To investigate the structural characteristics of bulk LSM and YSZ as well as their free surfaces and LSM/YSZ interfaces, periodic supercells (for the bulk) and periodic slabs (for surfaces and interfaces) were constructed with different local structures and with and without bulk and surface defects (vacancies, impurities, substitutional defects, etc.). All of the slabs were at least five layers thick (for LSM/YSZ interfaces, each of the touching LSM and YSZ slabs were at least five layers thick) to ensure relevant surface/interface relaxation. All of the considered geometries were optimized using DFT. The vacuum layer between periodically repeated slabs was $>15 \AA$, which is enough to avoid direct interactions between neighboring supercells. 
The positions of all atoms at one of the lower slab surfaces were fixed while all the other layers of the slab were allowed to relax. The surface defects of interest were constructed at the free (unfixed) surface; the bulk and/or subsurface defects were created somewhere within the slab but not in the "fixed" layer. The initial slabs were made by cutting off ideal bulk perovskite (for LSM) or fluorite (for cubic YSZ) structures. The optimal number of atomic layers for the slab calculations was chosen by comparing defect-formation energies for slabs with different numbers of layers (the number was increased until the energy difference for $n$ and $(n+1)$ layers is no larger than $0.2 \mathrm{eV}$, which is comparable with the error bar of calculations). An important fact is that point defect-formation energy as well as its migration barrier is usually defined by the local structure of the surrounding network, i.e., by 1-2 of the nearest neighboring layers of metal cations. Therefore, there was no need to perform a full systematic study of the statistics for all possible supercells with different topologies. Instead, different local structures around defects in the bulk and at the surface were created.

All calculations were based on the generalized gradient approximation (GGA) for exchange and correlation, and plane waves. ${ }^{30}$ The GGA functional of Perdew, Burke, and Ernzerhof (PBE) ${ }^{31}$ were used, which gives good results for chemisorption of molecules at transition-metal surfaces. Projected augmented wave (PAW) scalar relativistic pseudopotentials ${ }^{32}$ and the VASP code ${ }^{27,28,29}$ were used. The energy cutoff for the plane-wave basis was set at $500 \mathrm{eV}$, and all integrations over the Brillouin zone were done using the Monkhorst-Pack scheme with eight $\mathbf{k}$ points in the relevant irreducible wedge. ${ }^{33}$ Inclusion of additional $\mathbf{k}$ points was found to have minimal effect on the total-energy differences of interest here. The total number of atoms varied between 200 and 350 for different periodic supercells. For each supercell, we relaxed all atoms until the quantum-mechanical force on each atom became smaller than $0.02 \mathrm{eV} / \AA ̊$. All the calculations were spin-polarized. Activation barriers were calculated using the nudged-elastic-band method. ${ }^{34}$

The formation energies and migration barriers for several different defects in solid solutions corresponding to the materials and interfaces used in SOECs were calculated. In particular, the role of different point defects (oxygen vacancies, interstitials, substitutional defects, etc.) in degradation of bulk materials, surfaces, and interfaces and in the processes of transport and trapping of different atoms at interfaces were investigated. The values calculated from first-principles were used for calculating transport coefficients in KMC codes.

\subsubsection{Thermodynamics and Phase Equilibria}

One mechanism of the SOEC degradation might be related to a simple phase decomposition of the materials used for the SOEC electrodes, electrolyte, and interconnects (especially at different interfaces) because of the lack of thermodynamic stability at the operating conditions. This statement could be verified if systematic modeling (performed in a close collaboration with experimental work) were conducted on the construction of phase diagram isotherms and isopleths for all materials of interest. Self-consistent modeling of thermodynamic properties and phase equilibria of materials was conducted using the Thermo-Calc code - an equilibrium thermodynamics tool specifically developed for such applications. It is based on the classical thermodynamics of heterogeneous equilibria ${ }^{35,36,37}$ and was realized in Sweden. ${ }^{38}$ In order to make this program work, it is also necessary to provide it with thermodynamic and/or existing experimental phase equilibria data. These data come in the form of different proprietary and public databases. In those cases when the necessary information is absent, it needs to be generated using first-principles atomistic simulations. ${ }^{39}$

Thermo-Calc calculations are based on the principle of finding a global equilibrium among all possible competing phases at given conditions. The connection between phase diagrams and thermodynamic properties of materials is established via the principle of Gibbs' tangent plane construction: when it circumvents the "cups" of thermodynamic potentials of competing phases, the project of the traces of figurative points where the plane has actually touched the potential surfaces onto the "T- $\mathrm{x}$ " plane represents the corresponding phase diagram. 
The type of material degradation processes considered above is associated with complete loss of thermodynamic stability of materials, e.g., when the coefficients of chemical, mechanical, and thermal stability become equal to zero. An example of such phase transformation is spinodal decomposition. ${ }^{40}$ In this case, no external forces are required to cause material degradation. However, this is not the only mechanism that could be operable in an SOEC. Other mechanisms might include diffusion-induced phase transformations, when the diffusion of a certain chemical element into (or out of) material may result in phase transformations and material degradation.

\subsubsection{Diffusion Modeling}

Problems of this type could be handled with a computational tool called Dictra (Diffusion-Controlled Transformations). This is a sister software of the thermodynamic program "Thermo-Calc"; it was also developed at the Royal Institute of Technology in Stockholm, Sweden. This software was used, in application to the SOEC material degradation problem, to select a suitable coating material for the 441 ferritic steel interconnects, which may otherwise poison electrodes, electrolyte, and bond-layer materials by gaseous chromium species released from stainless steel materials at $800^{\circ} \mathrm{C}$ in the presence of water vapor.

Dictra code operates in the following way. ${ }^{41}$ After each diffusion step, the equilibrium corresponding to the new average composition is calculated at every spatial point inside the considered system, and the corresponding equilibrium calculations are made anew using the Thermo-Calc software. ${ }^{42}$ This procedure ensures that all composition and phase-fraction profiles are updated. The diffusion step is then repeated with the new composition profiles in the matrix, and so on. It should be understood that the dispersed particles will affect the diffusivity through the material; this problem could be treated separately if necessary. $^{43}$

It is very important to mention that Dictra works in tandem with Thermo-Calc; under the assumption of local equilibrium at every step, it computes the chemical potential gradients (second derivatives of the Gibbs potential) and makes a one-time step forward. Consequently, it needs data on equilibrium thermodynamics of systems under consideration, as well as the corresponding atomic mobilities. For steels, both types of databases are readily available and were acquired along with the computational tools. However, for oxides and perovskites (the use of these materials is critically important for the SOEC functionality), any mobility databases are absent and need to be developed. This should be done using first-principles atomistic calculations and atomic-scale modeling. In particular, the diffusivity matrix should be constructed by combined efforts of searching the existing literature on oxides and perovskites as well as by performing atomic-scale modeling (MD and KMC) for these ceramics. Kinetic modeling will also help to optimize the materials and coatings by minimizing all undesirable transport processes (e.g., Cr transport from steel interconnects) in the SOEC systems.

Combined atomic-scale, thermodynamic, and kinetic modeling will eventually provide practical recommendations on how to modify the SOEC multi-component systems to minimize Cr transport, oxygen delamination, and other undesirable processes, thus increasing the lifetime of SOEC devices and making it possible to use them in industrial and/or commercial applications.

\subsection{Results and Discussion}

\subsubsection{Phase Equilibria}

Mechanisms of the SOEC materials (electrodes, electrolytes, and interconnects) degradation are ubiquitous and include cascades of different reactions of phase decomposition (eutectoid reactions, spinodal decomposition) and the formation of new a multi-component phase (peritectoid reactions, congruent formation of stoichiometric compounds and solid solubility ranges based upon them). Since 
modern thermodynamic databases for such systems are absent, the phase-equilibrium modeling could not be executed, and the detailed analysis of existing data on phase equilibria were used. While this approach does not exhaust all possibilities of the formation of quaternary, quinary, and higher-order compounds, it nevertheless reveals an astounding picture of the cascades of different phase transformations even in such simplified systems that would, undoubtedly, result in the degradation of the SOEC systems with time. This, however cursory, analysis of phase equilibria in the SOEC materials convinces us that while some palliative measures (coatings, protective interlayers of $\mathrm{CeO}_{2}$ etc.) could be undertaken with some degree of success, it is not sufficient to solve the underpinning major engineering problem: how to make such devices work reliably for some 20,000 hours or more. More attention should be paid not to the improvement of the materials base of such a system (it has been quite developed already), but rather to the SOEC process control.

SOECs degradation issues are closely related to diffusion of atoms between the device components. At SOEC operating temperatures $\left(\sim 800^{\circ} \mathrm{C}\right)$, these diffusion processes become operable, and different atoms migrate across interfaces, penetrate into initially pristine materials, form new phases, and modify the device's functionality. Recent post-test examinations of the existing SOEC devices indicated that transport of Cr-containing species from steel interconnects into the oxygen electrode and lanthanum strontium cobalt (LSC) bond layer and cation segregation and phase separation in the bond layer provide a major contribution into the overall SOEC degradation. ${ }^{23}$

First-principles calculations and atomic-scale modeling for defects in different oxides and ceramics indicate that atomic migration processes can significantly affect the local crystal structure of the material. In particular, migrating hydrogen species in alumina may accumulate in some spatial regions (typically, around aluminum vacancies), break surrounding inter-atomic bonds, and eventually, create large voids in alumina. ${ }^{44}$ Such a phenomenon eventually will cause mechanical degradation (breakdown) of the alumina film that protects aluminum metal from an aggressive chemical environment and initiates pitting corrosion. It was also demonstrated that hydrogen atoms can combine with vacancies in bulk $\mathrm{Al}$ and play a crucial role in the embrittlement of this prototypical ductile solid. ${ }^{45}$

Recent experiments confirm that similar diffusion-related phenomena should occur in different components of the SOEC devices, namely, Zr penetrates into the LSM material while La and Mn penetrate into the YSZ electrolyte due to diffusion across a LSM/YSZ interface. A presence of preexisting interfacial defects and granulated structure should even accelerate such a "mixing-up" process resulting in appearance of different new phases at the interface including $\mathrm{LaZrO}_{3}, \mathrm{SrZrO}_{3}, \mathrm{La}_{2} \mathrm{O}_{3}, \mathrm{Mn}_{2} \mathrm{O}_{3}, \mathrm{La}_{2} \mathrm{Zr}_{2} \mathrm{O}_{7}$, etc., which cause the decline of the functionality of both electrode and electrolyte. ${ }^{24}$

As mentioned above, the self-consistent data on thermodynamics and phase equilibria in the whole $\left\{\mathrm{ZrO}_{2}-\mathrm{Y}_{2} \mathrm{O}_{3}\right\}-\left\{\mathrm{La}_{2} \mathrm{O}_{3}-\mathrm{Mn}_{2} \mathrm{O}_{3}-\mathrm{SrO}\right\}$ quasi-quinary system are absent. The most extensive work on this subject was published by Chen et $\mathrm{al}^{46}$ The authors considered the $\mathrm{La}-\mathrm{Mn}-\mathrm{Y}-\mathrm{Zr}-\mathrm{O}$ system and assumed that ideal extrapolations could be made from the corresponding quasi-binary and ternary systems to quaternary and then to the quinary $\mathrm{La}-\mathrm{Mn}-\mathrm{Y}-\mathrm{Zr}-\mathrm{O}$ system. Such an extrapolation scheme has deficiencies. For example, the formation of any new compounds (comprising more than 3 elements) turns out to be impossible; this was confirmed by a number of phase diagram projections obtained using the CALPHAD method.

On the other hand, the existence of more complex compounds in these systems was confirmed experimentally. For example, Mitterdorfer and Gauckler observed, among other things, heavy $\mathrm{Zr}$ diffusion from YSZ into quaternary Mn-deficient compounds: $\mathrm{La}_{0.85} \mathrm{Sr}_{0.15} \mathrm{Mn}_{0.95} \mathrm{O}_{3}$ and $\mathrm{La}_{0.85} \mathrm{Sr}_{0.15} \mathrm{Mn}_{0.98} \mathrm{O}_{3}{ }^{47}$ Because at present, it is impossible to analyze the YSZ -LSM diffusion couple in its entirety, quasi-binary and ternary systems were analyzed as follows: $\mathrm{ZrO}_{2}-\mathrm{SrO} ; \mathrm{ZrO}_{2}-\mathrm{Mn}_{2} \mathrm{O}_{3} ; \mathrm{ZrO}_{2}$ $\mathrm{La}_{2} \mathrm{O}_{3} ; \mathrm{Y}_{2} \mathrm{O}_{3}-\mathrm{La}_{2} \mathrm{O}_{3} ; \mathrm{Y}_{2} \mathrm{O}_{3}-\mathrm{Mn}_{2} \mathrm{O}_{3}$; and $\mathrm{Y}_{2} \mathrm{O}_{3}$-SrO. 
An isothermal cross-section of the $\mathrm{Zr}-\mathrm{Sr}$-O ternary phase diagram at $1000 \mathrm{~K}$ (i.e., at a temperature which is quite close to the typical SOEC device operational temperatures) shows that, depending upon the ratio of $\mathrm{ZrO}_{2}$ and $\mathrm{SrO}$ in this system, one could expect the formation of a diffusion couple, in which such compounds (phases) as $\mathrm{Sr}_{2} \mathrm{ZrO}_{4}, \mathrm{Sr}_{3} \mathrm{Zr}_{2} \mathrm{O}_{7}, \mathrm{Sr}_{3} \mathrm{Zr}_{4} \mathrm{O}_{10}$, and $\mathrm{SrZrO}_{3}$ will be observed as the concentration of $\mathrm{Sr}$ in $\mathrm{ZrO}_{2}$ keeps growing with time. ${ }^{48,49}$ Depending upon the conditions of SOEC operation, these compounds could be observed at progressively larger distances from the $\mathrm{ZrO}_{2}-\mathrm{SrO}$ interface ( $\mathrm{Sr}$ diffuses into the bulk of $\mathrm{ZrO}_{2}$ ). It is the formation of such cascades of compounds that makes their reliable detection quite difficult, and at the same time it is very detrimental to the electrical conductivity of such an interface.

In Reference 50, a portion of the $\mathrm{ZrO}_{2}-\mathrm{La}_{2} \mathrm{O}_{3}$ system has been studied corresponding to the concentration range $\mathrm{ZrO}_{2}-\mathrm{La}_{18.2} \mathrm{O}_{63.6} \mathrm{Zr}_{18.2}$. It was established that in the $500-1700^{\circ} \mathrm{C}$ temperature range, a non-stoichiometric compound, $\mathrm{La}_{1.64} \mathrm{Zr}_{0.27} \mathrm{O}_{3}$, is formed. In addition, the existence of another ternary compound, $\mathrm{La}_{2} \mathrm{Zr}_{2} \mathrm{O}_{7}$, is also described in the literature. ${ }^{47}$

$\mathrm{MnO}_{\mathrm{x}}$ has some solubility in the cubic $\mathrm{ZrO}_{2}$ phase, but in the presence of yttrium may form hexagonal $\mathrm{YMnO}_{3}$ as well as a single crystal with a formula of $\mathrm{Y}_{0.95} \mathrm{Zr}_{0.05} \mathrm{MnO}_{3}{ }^{51}$

The $\mathrm{La}_{2} \mathrm{O}_{3}-\mathrm{Y}_{2} \mathrm{O}_{3}$ displays a number of high-temperature phase transformations between these two compounds. In the temperature range of SOECs, the existence of a stoichiometric compound $\mathrm{LaYO}_{3}$ that is formed incongruently at $1578^{\circ} \mathrm{C}^{52}$ should be of interest.

The isothermal cross-section of the La-Mn-O phase diagram at $800^{\circ} \mathrm{C}$ was constructed by Grundy et al. ${ }^{53}$ According to Reference 49 , there is a significant solubility range on the basis of a non-stoichiometric compound, $\mathrm{La}_{0.95} \mathrm{Mn}_{0.95} \mathrm{O}_{3}$.

Finally, an isothermal cross-section of the La-Sr-O phase diagram at $900^{\circ} \mathrm{C}$ was constructed by Jacob and Waseda, ${ }^{54}$ who discovered the formation of a ternary compound of the spinel type, $\mathrm{SrY}_{2} \mathrm{O}_{4}$.

Summing up the results reviewed in this section, one comes to a rather pessimistic conclusion about the SOEC material design resources, which seem to have been practically exhausted by now. Most probably, the hope to find systems that would be thermodynamically stable and not undergo any undesired phase transformation is unrealistic. Consequently, the problem becomes that of diffusion stability of materials and their kinetic survivability. Perhaps, a better chance of success lies in trying to optimize not only the diffusion processes and control-phase transformations in different SOEC materials, but the electrochemical regime of the device as a whole.

\subsubsection{Structural Models}

In both SOEC and SOFC devices, the LSM electrode is porous while the YSZ electrolyte is dense. Therefore, at their interface, oxygen gas accumulated in porous LSM material should be in dynamic equilibrium with free electrolyte surface, which affects the vacancy concentration in YSZ. Therefore, it is natural to start atomic-scale modeling of the processes at the oxygen electrode-electrolyte interface considering two structural models - a free YSZ surface (we consider just a cubic $\mathrm{ZrO}_{2}$ phase) and an $\mathrm{LSM} / \mathrm{YSZ}$ interface.

LSM was widely used in SOFC devices because its thermal expansion coefficient is well-matched with that of YSZ, a common material for SOFC electrolytes. The low-temperature $\mathrm{LaMnO}_{3}$ structure is orthorhombic (comprising four formula units, space group Pbnm) ${ }^{55}$ Neglect of the effects of the Jahn-Teller distortion and mutual tilting and rotation of $\mathrm{MnO}_{6}$ octahedra transforms the orthorhombic structure into the tetragonal one. ${ }^{56}$ At high temperatures (above $750 \mathrm{~K}$ ), $\mathrm{LaMnO}_{3}$ transforms into the ideal perovskite structure (space group $\mathrm{Pm} 3 \mathrm{~m}$ ) with five atoms (one formula unit) per primitive cubic unit cell, with the experimental lattice constant $a=3.947 \AA$ [56]. First-principles calculations gave $3.96 \AA$ for the $\mathrm{LaMnO}_{3}$ cubic phase, in good agreement with this value. 
Pure $\mathrm{ZrO}_{2}$ displays three polymorphs at atmospheric pressure: cubic (c-), tetragonal (t-), and monoclinic (m-). ${ }^{57}$ At ambient pressure, the ground-state $\mathrm{ZrO}_{2}$ has a monoclinic structure up to a temperature of $1450 \mathrm{~K}^{58}$ when the $\mathrm{m}$ - to t-phase transition occurs accompanied by a decrease in unit-cell volume. The cubic fluorite phase of zirconia is only thermodynamically stable at temperatures higher than $2650 \mathrm{~K}$. A simple explanation of this fact is that $\mathrm{Zr}^{4+}$ ions appear to be too large for an efficiently packed rutile structure and too small to form the fluorite structure. ${ }^{59}$ Stabilization of $\mathrm{c}-\mathrm{ZrO}_{2}$ (which is especially important in high-temperature applications where cycling occurs through the $\mathrm{m}$ - $\mathrm{t}$ transition temperature during material preparation or use) down to room temperature can be achieved by doping with $\mathrm{Y}^{3+60}$ which forms the YSZ ceramics used in practical applications. In our calculations we consider just a cubic $\mathrm{ZrO}_{2}$ phase, i.e., it is implied that it is always stabilized by adding a sufficient quantity of yttrium ions. The lattice constant for this structure obtained from first-principles, self-consistent electronic structure calculations is $5.17 \AA$ (corresponding to the $\mathrm{Zr}$ - Zr distance of $3.65 \AA$ A between two nearest Zr neighbors), in good agreement with the experimental value of $5.07 \AA .^{61}$

\section{a)}

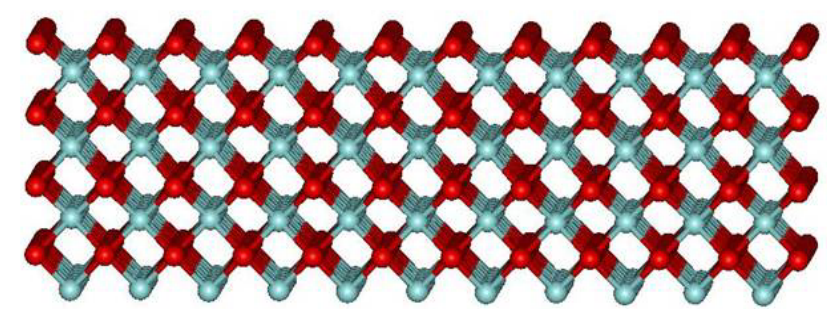

b)

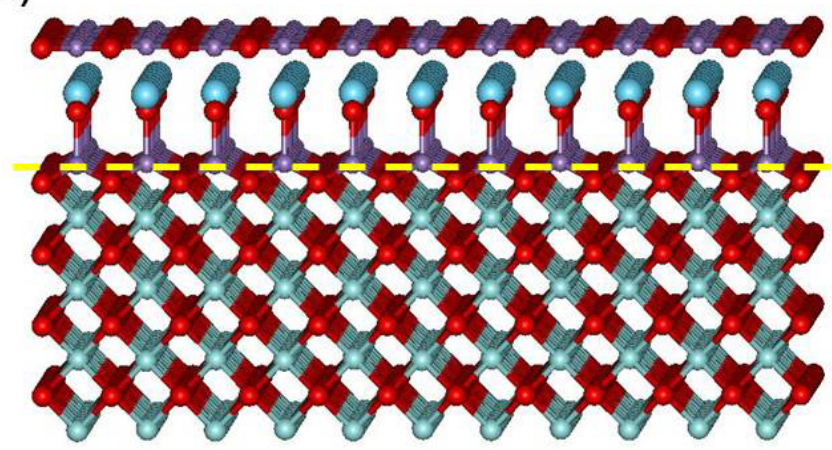

Figure 4. Atomic-scale models for YSZ surface and LSM/YSZ interface: (a) a free (001) surface for the oxygen-saturated stabilized cubic $\mathrm{ZrO}_{2}$ structure; (b) a smooth $\mathrm{LaMnO}_{3} / \mathrm{ZrO}_{2}$ interface with (001) orientation. Oxygen atoms are shown in red, $\mathrm{Zr}$ in small light blue balls, La in big cyan balls, Mn in purple. Only three layers of $\mathrm{LaMnO}_{3}$ perovskite (nearest to the interface) are shown.

Calculations also indicated that these two materials may form high-quality interfaces. Figure $4 \mathrm{~b}$ shows the relaxed structure of the $\mathrm{LaMnO}_{3} / \mathrm{ZrO}_{2}$ interface obtained by positioning a (001) surface of $\mathrm{LaMnO}_{3}$ on top of the oxygen-saturated (001) surface of cubic $\mathrm{ZrO}_{2}$ (the interfacial oxygen atoms were common for the $\mathrm{ZrO}_{2}$ and $\mathrm{MnO}_{2}$ layers, i.e., some "spare" surface oxygen atoms were eliminated). Only the positions of zirconium and oxygen atoms in the lower $\mathrm{ZrO}_{2}$ layer were fixed while all the other atoms were allowed to relax. Figure $4 \mathrm{~b}$ clearly indicates that the interfacial $\mathrm{MnO}_{2}$ layer (in which $\mathrm{Mn}$ atoms should share oxygen atoms with $\mathrm{Zr}$ atoms) is very corrugated in comparison with perovskite $\mathrm{MnO}_{2}$ layer; oxygen atoms in the $\mathrm{LaO}$ layer are shifted down towards the interfacial $\mathrm{Mn}$ atoms while the next $\mathrm{MnO}_{2}$ layer is nearly flat as in an ideal perovskite structure. It means that only two interfacial layers of the $\mathrm{LaMnO}_{3}$ perovskite are affected by surface relaxation. In these calculations the perovskite structure was compressed (the lattice parameters of 3.65 Á corresponding to the $\mathrm{Zr}$ - $\mathrm{Zr}$ nearest-neighbor distance was 
taken, which is about $8 \%$ larger than the lattice parameter of $\mathrm{LaMnO}_{3}$ structure). However, the calculations indicate that even the second (from the interface) relaxed $\mathrm{MnO}_{2}$ layer is not significantly corrugated, i.e., the lattice parameter of $\mathrm{ZrO}_{2}$ governs the quality of the grown interface. Because of the latter parameter mismatch, one could expect that different interfacial points and extended defects (stacking faults, steps, dislocations, etc.) could be formed at the interface (especially at high temperatures). These defects could accumulate formed oxygen molecules (after $\mathrm{O}^{2-}$ ions leave the electrolyte and reduce to neutral $\mathrm{O}$ atoms, with subsequent $\mathrm{O}_{2}$ molecules formation) and, therefore, serve as precipitates for the formation of cracks that will result in the $\mathrm{O}_{2}$ delamination. However, calculations for the $\mathrm{LaMnO}_{3} / \mathrm{ZrO}_{2}$ interface indicate that mismatched defects could be formed in the bulk of $\mathrm{LaMnO}_{3}$ perovskite rather than at the interface, i.e., delamination also may occur in other perovskite layers.

\subsubsection{The Role of Dopants}

First-principles calculations were performed for the formation energies and migration barriers for $\mathrm{Y}$ atoms migrating to a free YSZ surface as well as for different atoms (Y, Zr, La, Mn, Sr, O) migrating across the LSM/YSZ interface. Such a migration at operating temperatures of SOEC and SOFC systems is a well-known fact that has been investigated for SOFCs in the past. ${ }^{24,62}$ Calculations were performed for different possible interstitial and substitutional defects near a free YSZ surface (Figure 2) and near LSM/YSZ interface (Figure 4). Typically, substitutional defects for metal impurities in complex oxides have much lower total energies than interstitials. However, at high temperatures one could expect that a significant number of oxygen vacancies in both LSM and YSZ materials will be formed. Therefore, many other low-energy atomic configurations with different local structures are possible. Also, many additional migration paths for metal atoms will be available.

Figure 5 shows the schematics of the structural changes for three substitutional defects (Y, Mn, and La impurities substituting "regular" Zr atoms) positioned near a free YSZ surface. In order to keep the valence balance of the considered supercells, one oxygen vacancy was added for each pair of substituted 4 -valent $\mathrm{Zr}$ by 3-valent impurity atoms $\left(\mathrm{Y}^{3+}, \mathrm{Mn}^{3+}\right.$, or $\left.\mathrm{La}^{3+}\right)$. Therefore, an even number of such defects were used. The results indicate that substituting two surface or subsurface (one or two layers below the surface) $\mathrm{Zr}$ atoms by $\mathrm{Y}$ and $\mathrm{Mn}$ dopants does not produce any significant damage to a free electrolyte surface and does not reconfigure the surface. La surface or subsurface substitutional dopants do produce a significant damage that reconfigures the surface. This reconfiguration may significantly change the oxygen network near the surface (e.g., facilitate the formation of the O-O peroxy bonds at the surface). Then the formation of free $\mathrm{O}_{2}$ molecules at the surface may become energetically favorable, i.e., these molecules may leave the electrolyte surface as well as accumulate in different structural defects (e.g., preexisting subsurface voids), which may cause void growth and crack initiation.

Similar calculations were performed for substitutional defects near the LSM/YSZ interface (Figure 5). It was found that $\mathrm{La}$ and $\mathrm{Sr}$ metal impurities migrating across the interface into the electrolyte may significantly change the YSZ local structure near the interface (substitution of one $\mathrm{Zr}^{4+}$ by $\mathrm{Sr}^{2+}$ requires one oxygen vacancy to be introduced in the supercell). In particular, these large atoms generate a significantly larger distortion in the electrolyte network than Mn impurities simply because the La-O and $\mathrm{Sr}-\mathrm{O}$ atomic bonds are longer than the $\mathrm{Zr}-\mathrm{O}$ and $\mathrm{Mn}-\mathrm{O}$ bonds that cause increased local strain in the electrolyte network. This explains why La penetration into YSZ develops much slower than Mn migration across the interface. ${ }^{62}$ 
a)

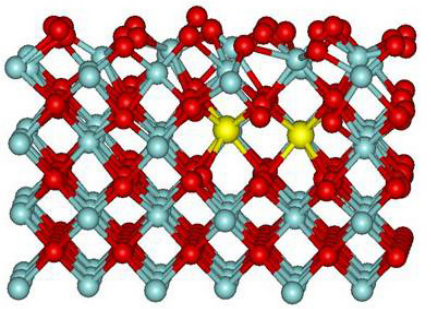

b)

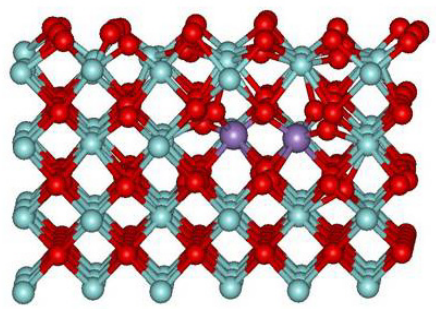

c)

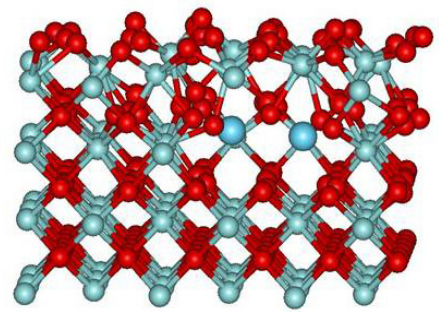

Figure 5. Free YSZ (001) surface, schematics of Y (a), Mn (b), and La (c) subsurface substitutional defects. In each case, two subsurface $\mathrm{Zr}$ atoms are substituted by dopant impurity atoms and one oxygen vacancy is added. Oxygen atoms are shown in red, $\mathrm{Zr}$ in small light blue balls, Y in yellow, La in big cyan balls, Mn in purple.

An accumulation of a significant number of $\mathrm{La}$ and/or $\mathrm{Sr}$ impurities at the interfacial region may generate the conditions for the formation of oxygen O-O peroxy bonds. The latter are well-established precursors of oxygen molecules that will accumulate within strained regions or voids and subsequently initiate delamination cracks. Pre-existing defects in the bulk of the electrolyte and at the interface between the electrolyte and porous perovskite electrode (oxygen vacancies, voids, grain boundaries, interfacial stacking faults, etc.) may facilitate the La migration and, consequently, make the onset of delamination much easier.

Although Mn dopants do not produce any significant damage to the LSM/YSZ interface (like La and $\mathrm{Sr}$ atoms, see Figure $6 \mathrm{c}$ ), $\mathrm{Mn}$ is mixed valence transition metal, and its accumulation within the electrolyte in the vicinity of the interface may significantly increase the electronic conductivity (e.g., through the $\mathrm{Mn}^{3+}-\mathrm{Mn}^{4+}$ transitions). ${ }^{63}$ A similar mechanism of electronic conductivity (related to $\mathrm{Ce}^{3+}$ $\mathrm{Ce}^{4+}$ transitions) was observed in ceria-doped $\mathrm{YSZ}^{64}$ This phenomenon may cause a premature conversion of oxygen ions $\mathrm{O}^{2-}$ to neutral oxygen atoms in the electrolyte, and subsequently, into $\mathrm{O}_{2}$ molecules that may be accumulated within voids and other defects, develop pressure, and, eventually, accelerate crack formation.

Therefore, our calculations showed that migration of La, Sr, and Mn atoms from porous LSM anode into YSZ electrolyte may cause a significant damage at the interface between these materials and initiate the $\mathrm{O}_{2}$-delamination process. Although $\mathrm{La}, \mathrm{Sr}$, and $\mathrm{Mn}$ atoms behave differently, all these impurities may participate in degradation mechanisms. Most likely, degradation occurs as a collective effect of all the atoms' mixing-up processes at the interface, and inhibition (or mitigation) of migration of all these elements across the LSM/YSZ interface is needed to improve the performance of solid-state devices. 
a)

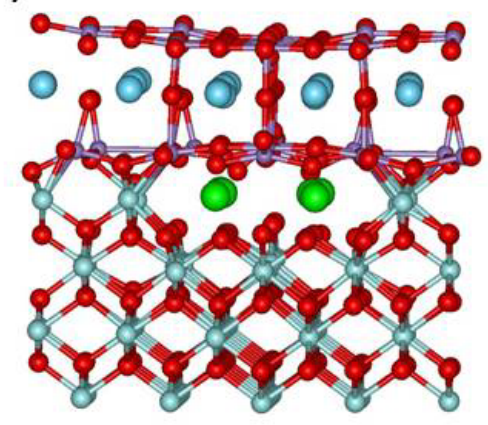

c)

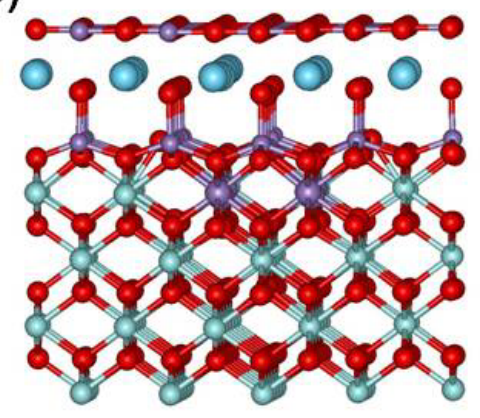

b)

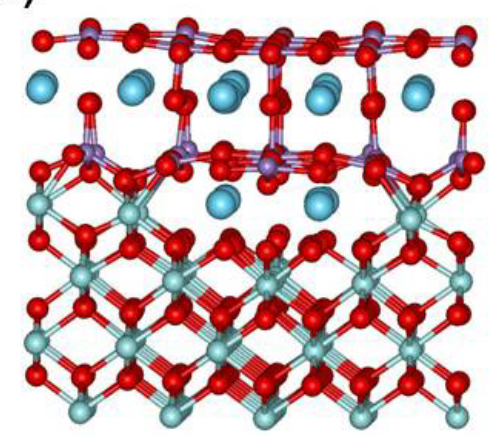

d)

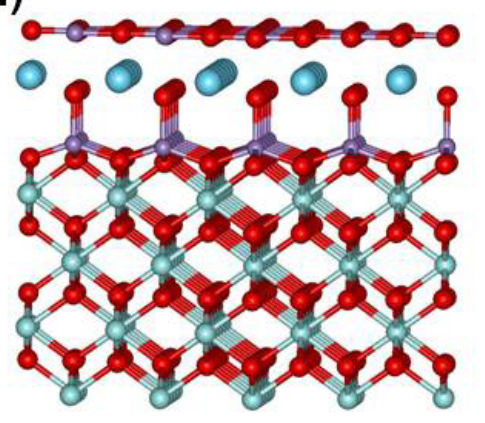

Figure 6. Schematics for substitutional $\mathrm{Sr}(\mathrm{a})$, and $\mathrm{La} \mathrm{(b),} \mathrm{and} \mathrm{Mn}$ (c) impurity defects in subsurface area of YSZ near the LSM/YSZ interface. A whole row of Zr atoms in the supercell was substituted by impurities. A defect-free interface is shown for comparison (d). Oxygen atoms are shown in red, $\mathrm{Zr}$ in small light blue balls, La in big cyan balls, $\mathrm{Mn}$ in purple, $\mathrm{Sr}$ in green. Only three layers of $\mathrm{LaMnO}_{3}$ perovskite (nearest to the interface) are shown. $\mathrm{La}-\mathrm{O}$ and $\mathrm{Sr}-\mathrm{O}$ bonds were omitted in figures (a) and (b) for better illustration.

\subsubsection{Molecular Oxygen Formation and Interfacial Pressure Development}

Oxygen ions $\left(\mathrm{O}^{2-}\right)$ migrate through the electrolyte as charged particles, and neutral oxygen species are formed in the oxygen electrode or at the oxygen/electrolyte interface. Electronic conductivity plays an important role in this process because ions should get rid of their charge and convert to neutral species, i.e., electronic current provides a sink for this extra charge. Due to the high porosity of the oxygen electrode, oxygen molecules may be formed in the areas of diffuse LSM/YSZ interface where a free YSZ surface is exposed to one of preexistent LSM pores, i.e., at a free YSZ surface. When the surface is oxygen saturated, an $\mathrm{O}_{2}$ molecule formation may be initiated by a formation of the O-O peroxy bond with subsequent release of a free molecule. At a pure cubic zirconia surface, peroxy bonds could be formed due to surface oxygen migration when an extra oxygen atom goes into a preexisting $\mathrm{Zr}$-O-Zr surface bond. When some surface or subsurface 4-valent $\mathrm{Zr}$ atoms are substituted by lower valence dopants (3-valent $\mathrm{Y}, \mathrm{La}$, or $\mathrm{Mn}$, and 2-valent $\mathrm{Sr}$ ), peroxy bonds could be formed by surface relaxation and are energetically favorable (Figure 7a). Such a surface O-O bond formation simply reflects the fact that the system has a tendency to reach valence balance and get rid of some oxygen atoms by forming a corresponding number of vacancies at the surface of subsurface layers.

In order to estimate the oxygen gas pressure in a preexisting interfacial void, a simple thermodynamic equation was used for the change of the pressure- and temperature-dependent Gibbs potential at the free YSZ surface (or at a free surface within a void in the bulk of the electrolyte): 


$$
\Delta G(p, T)=E^{\text {surface-2O } O^{2-}}-E_{m o l}^{O_{2}}-E^{\text {surface }}+\mu_{O_{2}}^{(0)}+k T \cdot \ln \frac{p_{O_{2}}}{p_{O_{2}}^{(0)}}
$$

where the first three terms in the right hand side of the equation correspond to the $\mathrm{O}_{2}$ formation energy at the surface, $E_{\mathrm{O}_{2}}^{f} ; \mu_{\mathrm{O}_{2}}^{(0)}$ and $p_{\mathrm{O}_{2}}^{(0)}$ are oxygen chemical potential and pressure at "normal conditions"

( $1 \mathrm{~atm}$; in practical calculations, $\mu_{\mathrm{O}_{2}}^{(0)}=0$ ), and $p_{\mathrm{O}_{2}}$ is the partial pressure of the $\mathrm{O}_{2}$ gas, which is in an equilibrium condition with the surface of the electrolyte. In a reality, the situation is more complicated, and the characteristic time of the migration of oxygen species and metal dopants in the interfacial region as well as the kinetic barriers for $\mathrm{O}_{2}$ formation should be taken into account to understand the characteristic growth time for the $\mathrm{O}_{2}$ - filled voids. However, such a simple thermodynamics model could provide a good starting point for catching some important features of these systems.

a)

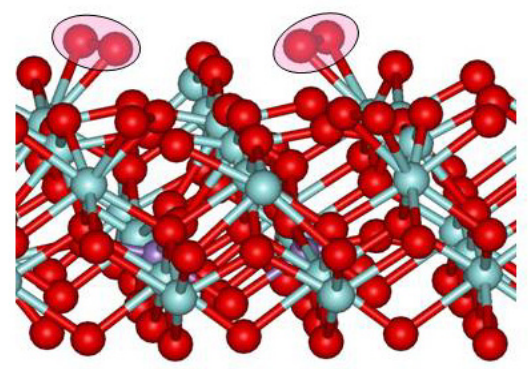

b)

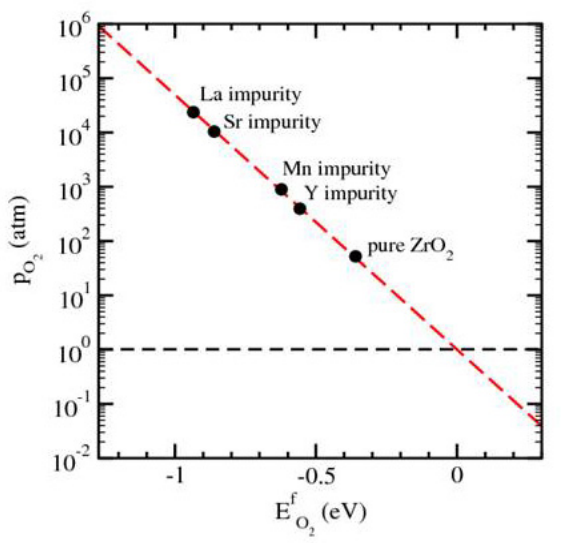

Figure 7. (a) Schematics of the formation of peroxy O-O bonds (precursors of $\mathrm{O}_{2}$ molecules) at the (001) surface of cubic $\mathrm{ZrO}_{2}$ related to substitutional $\mathrm{Mn}$ impurities in the subsurface region. Oxygen atoms are shown in red, $\mathrm{Zr}$ - as small light blue balls, $\mathrm{Mn}$ - in purple; (b) Equilibrium pressure of $\mathrm{O}_{2}$ gas above a free $\mathrm{ZrO}_{2}$ surface at $T=800^{\circ} \mathrm{C}$ as a function of $\mathrm{O}_{2}$ formation energy corresponding to different subsurface substitutional defects. 
First-principles calculations indicate that the surface of the electrolyte is intrinsically unstable (the $\mathrm{O}_{2}$ formation energy is negative, i.e., formation of $\mathrm{O}_{2}$ molecules is energetically favorable, see Figure 7b), and low-valence impurities positioned into the subsurface region increase the surface instability even further (the $\mathrm{O}_{2}$ formation energy becomes more negative in comparison with pure cubic $\mathrm{ZrO}_{2}$ surface with [001] orientation). In these calculations, two $\mathrm{Zr}$ atoms in the subsurface layer were substituted by two 3-valent (Y, La, Mn) impurities (or one $\mathrm{Zr}$ atom by one 2-valent $\mathrm{Sr}$ impurity) and calculated the $\mathrm{O}_{2}$ formation energy for surface $\mathrm{O}$ atoms positioned close to these substitutional defects (e.g., in the peroxy bonds formed during surface relaxation, Figure 7a). This result means that the YSZ surface always has a tendency to evaporate oxygen gas and form oxygen vacancies at the surface that later may drift into the bulk. This phenomenon is not surprising - otherwise, this material could not exhibit good oxygen ion diffusion that occurs through a vacancy mechanism.

There is another interesting result that follows from Equation (1) and Figure 7b. Assume that oxygen gas evaporated from the $\mathrm{ZrO}_{2}$ surface is accumulated within some cavity inside the electrolyte (void, pore, crack, etc.) or in a LSM pore at a LSM/YSZ interface. Because of negativity of the $\mathrm{O}_{2}$ formation energy in all of the system being considered is negative, oxygen gas evaporation will stop (and the equilibrium reached) when the pressure term in Equation (1) will make $\Delta G$ positive (Figure $7 \mathrm{~b}$, vertical axis). It means that equilibrium oxygen gas concentration within the void is controlled by the gas pressure within the void, which is defined by the structure and chemical composition of the void surface. Here we assumed that the void is closed, i.e., formed oxygen gas cannot flow away from the void. Closed voids should be the main type of extended defects where gas pressure could be developed and cause the void growth and crack formation. Figure $7 \mathrm{~b}$ clearly indicates that the presence of $\mathrm{La}$ and $\mathrm{Sr}$ impurities at the internal surfaces of closed voids can lift the equilibrium pressure of oxygen gas by several orders of magnitude in comparison with pure $\mathrm{ZrO}_{2}$ or YSZ.

However, the formation of neutral oxygen gas molecules is possible only if the $\mathrm{O}^{2-}$ ion loses its two electrons and becomes neutral, i.e., the regions where $\mathrm{O}_{2}$ molecules are created should exhibit electronic conductivity in order to move these electrons away from charged oxygen ions. Therefore, we should not expect that neutral oxygen gas will be accumulated in closed voids inside undoped YSZ electrolytethere is no place for oxygen ions to lose their electrons without accumulating electric charge. However, the formation of oxygen gas is possible in voids below a "free" electrolyte surface if YSZ is doped with Mn (which increases the electronic conductivity) and/or at a closed LSM pore near the LSM/YSZ interface, which always contains a lot of different structural defects and grain boundaries that exhibit electron conductivity. In these conditions, a high pressure of $\mathrm{O}_{2}$ gas inside the void or pore may be reached, and the preexisting void with a characteristic size above some critical value will tend to grow spontaneously, i.e., the $\mathrm{O}_{2}$ delamination and crack formation processes will be initiated.

For a disk-shaped void, the force acting on both surfaces of the disk is $\sim p \cdot S$ where $p$ is the gas pressure within the disk, $S$ - its surface area. The disk starts to grow when the atomic bonding forces along the disk edge (proportional to $2 \pi R, R$ is the radius) are less than $p \cdot S$, and the bonds along the disk perimeter start to break. The critical radius $R_{c}$ could be approximately estimated as:

$$
R_{c} \sim \frac{E_{b}}{p \cdot l \cdot b}
$$

where $E_{b}$ is the binding energy of a single breaking bond, $l$ is the an average distance between neighboring breaking bonds (arranged along the perimeter of the disk edge), $b$ is the bond length of the breaking bond ( $\mathrm{Zr}-\mathrm{O}$ in our case). Simple estimates that used parameters obtained from first-principles calculations give the critical radius for a disk-shaped void on the order of $10 \AA$ for equilibrium $\mathrm{O}_{2}$ pressure of $p \sim 10^{5} \mathrm{~atm}$, which looks quite realistic for a closed void or pore (Figure 7b). It means that in doped YSZ, the void with the size of just several $\mathrm{Zr}-\mathrm{O}$ bond lengths may start to grow. 
The qualitative arguments considered above provided us with an opportunity to compare the two types of devices - SOEC and SOFC. In SOFC devices, a porous LSM oxygen electrode serves as a cathode, and the $\mathrm{O}_{2}$ gas comes into this electrode from the outside, i.e., the oxygen pressure in the electrode is maintained by this flow. In SOEC devices, the LSM electrode is an anode, and it collects only those oxygen species that came through the YSZ electrolyte. Therefore, one could expect a higher partial $\mathrm{O}_{2}$ pressure in the LSM pores near the LSM/YSZ interface in SOFCs than in SOECs. According to Equation (1), the higher oxygen gas pressure will inhibit the oxygen vacancy formation process in the electrolyte, and the lower concentration of $\mathrm{O}$ vacancies in $\mathrm{YSZ}$ will slow down the diffusion of $\mathrm{Mn}, \mathrm{La}$, and $\mathrm{Sr}$ into the electrolyte from the LSM electrode. Such a qualitative argument may explain why the $\mathrm{O}_{2}$ delamination processes in the SOFC devices are developing slower than in the SOEC devices, i.e., why the SOFC devices have a longer lifetime.

\subsubsection{Improving operation of SOEC devices by interface modifications}

A natural way of mitigation and/or slowing down the degradation mechanisms described above is to stop the undesirable migration of atoms between different SOEC components. In particular, it would be desirable to put an additional thin layer between the YSM electrolyte and LSM anode that would slow down the migration of $\mathrm{La}, \mathrm{Mn}, \mathrm{Sr}$, and $\mathrm{Zr}$ across the interface without reducing the oxygen transport. Several complex oxides may be considered as candidates for such a film - the characteristics that may be crucial for a choice of this material are: (i) the lattice constants should match both YSM and LSM crystal structure; (ii) the thermal expansion coefficients should be compatible; (iii) the tendency to form interfacial defects should be minimal; (iv) the diffusivity of $\mathrm{La}, \mathrm{Mn}, \mathrm{Sr}$, and $\mathrm{Zr}$ atoms through this material should be low.

As mentioned above, one of the main mechanisms of the electrolyte and oxygen electrode/electrolyte interface degradation is related to diffusion of relatively large $\mathrm{La}$ and $\mathrm{Sr}$ cations across the interface into the electrolyte. This process may form structural discontinuities resulting in deterioration of the SOEC properties. While the search for new and improved materials in this field continues, our preliminary calculations indicate that the degradation resistance of existing materials could be improved substantially using such techniques as coating and diffusion barriers. In the case of electrode/electrolyte (LSM/YSZ) interface, we think that a simple additional thin layer of $\mathrm{CeO}_{2-\mathrm{x}}$ oxide positioned between the LSM and YSZ layers should work very efficiently, preventing diffusion of large La and Sr cations.

Ceria is a well-known $\mathrm{O}^{2-}$ ion conductor and may be used as an electrolyte for SOFC devices itself. When the oxygen content $x$ of $\mathrm{CeO}_{2-\mathrm{x}}$ varies between 0 and 0.5 , ceria may be present in heterogeneous mixtures as a cascade of different unstable and metastable phases, and its ability to change the structure with variations of oxygen concentration is the key to understanding the ion conductivity mechanism in this material. In particular, ceria may be doped with other three-valent elements (e.g., gadolinium), which increases its conductivity due to formation of a larger number of oxygen vacancies similar to YSZ material. The cerium-gadolinium oxide, or CGO, with chemical formula $\mathrm{Ce}_{0.8} \mathrm{Gd}_{0.2} \mathrm{O}_{1.9}$, was shown to be a good barrier, arresting the diffusion of $\mathrm{Y}$ and $\mathrm{Zr}$ from the YSZ electrolyte and improving the SOFC performance. ${ }^{65}$ Actually, a 1.4- $\mu \mathrm{m}$ layer of CGO was sufficient to stop practically all diffusion. However, the CGO film used in these experiments was porous, and it may well happen that a better quality film may be even thinner. Other experiments on samaria-doped ceria (SDC) indicate that this film may stop the diffusion of Mo, $\mathrm{Mn}$, and $\mathrm{Sr}^{66}$

In order to better understand the diffusion of $\mathrm{La}$ and $\mathrm{Sr}$ cations across a ceria film, first-principles calculations and the NEB method ${ }^{35}$ were used to calculate migration barriers of La and Sr impurity across the ceria film. First, it was found that the self-diffusion migration barrier for $\mathrm{Ce}$ atoms in pure ceria is about $4.5 \mathrm{eV}$ (the diffusion occurs through the vacancy mechanism). Such a high diffusion barrier explains why no self-diffusion of Ce was experimentally observed at temperatures below $900^{\circ} \mathrm{C} .{ }^{66} \mathrm{The}$ migration of oxygen, however, starts at much lower temperatures $\left(\sim 500^{\circ} \mathrm{C}\right)$, which means that new 
mechanisms for Ce self-diffusion through ceria containing many oxygen vacancies may become operable. Anyway, one cannot expect any migration of $\mathrm{Ce}$ in ceria at typical SOEC working temperatures $\left(\sim 800^{\circ} \mathrm{C}\right)$.

For a substitutional La impurity defect, it was found from first-principles calculations that the migration barrier through ceria is even higher $(\sim 6.5 \mathrm{eV})$. Therefore, one cannot expect any significant migration of La across the ceria barrier layer even at $800^{\circ} \mathrm{C}$ as well. Indeed, a substitutional La defect produces a significant distortion of the local crystal structure in ceria by pushing surrounding oxygen atoms away, and it may be expected that migration of such a big cation will be suppressed. Such a behavior of La cation in ceria is very similar to that of lanthanum in $\gamma$-alumina described by Wang et al. ${ }^{67}$ La never goes into the bulk of the aluminum oxide and stabilizes its surface by attaching to it with high binding energy, which prevents any surface reconstructions and improves the catalytic behavior of $\gamma$--alumina (often used as a support for catalytic nanoparticles). Therefore, a ceria barrier between the YSZ electrolyte and LSM anode may be a good option to reduce the formation of oxide phases with high resistivity and to avoid oxygen delamination.

Another idea that naturally comes from the discussion in the previous section is to reduce the degradation processes at the LSM/YSZ interface leading to oxygen delamination by controlling the oxygen gas pressure in the porous SOEC anode. From Equation (1) it follows that increasing oxygen pressure (e.g., by providing an additional oxygen gas or air flow through the oxygen electrode) one could decrease the vacancy concentration in the regions of the electrolyte adjacent to the LSM/YSZ interface. This should slow down the migration of large cations (especially La and Sr) into the electrolyte and, therefore, increase the lifetime of the SOEC device. The complete understanding of these phenomena, however, necessitates more modeling efforts performed in close collaboration with experimental activities.

\subsection{Summary of Atomic-Scale Modeling}

In conclusion, an effort was made to identify, understand, and consequently propose different ways to mitigate oxygen delamination processes that reduce the lifetime of existing SOEC devices. The uniqueness of the approach is the use of a powerful combination of first-principles calculations and analysis of phase equilibria in order to understand the degradation mechanisms and provide practical recommendations on how to inhibit and/or completely mitigate them.

To continue this interdisciplinary modeling effort for the SOEC systems, there is a need to develop thermodynamics and mobility databases for the number of oxides. For some classes of materials (e.g., steels), both thermodynamic and mobility databases are readily available, while for oxides and perovskites used in the SOEC devices, mobility databases need to be developed. Because of insufficient experimental data for these materials, the only way to develop such databases is to use first-principles density functional calculations and atomic-scale modeling. The diffusivity matrices should be constructed by combined efforts of searching the existing literature on oxides and perovskites as well as by performing atomic-scale modeling (e.g., Molecular Dynamics) for these ceramics. Based on these databases, extended kinetic modeling will also help to optimize the real-life materials and coatings by minimizing all the undesirable transport processes (including $\mathrm{La}$ and $\mathrm{Sr}$ migration across the oxygen electrode-electrolyte interface) in the SOEC systems. 


\title{
3. NON-EQUILIBRIUM THERMODYNAMICS MODELING OF DEGRADATION IN SOLID OXIDE ELECTROLYSIS CELLS
}

\author{
(A. V. Virkar, University of Utah)
}

Earlier, Virkar $^{7}$ suggested that in a stack, cell-to-cell characteristics should be as uniform as possible so that, at a given operating current, the voltage across each cell is essentially the same. If, because of some structural/fabrication flaws, the cells are not identical, the resistance and voltage drop will vary from cell-to-cell. In such a case, the remainder of the cells in the stack will drive the cell with higher resistance. In an extreme case, for the stack to continue operating, the voltage across a cell with higher resistance can even become negative, which can eventually lead to cell failure and increased local temperatures. This phenomenon can spread to adjacent cells as a domino effect. In a normal SOFC, the (oxygen) ionic current is in a direction opposite to that of electronic current. However, if a cell has degraded to cause negative voltage, the direction of electron flow will reverse and both ionic and electronic currents will flow in the same direction. Based on this non-equilibrium thermodynamics phenomenon, Virkar ${ }^{22}$ developed a degradation model based on the premise that a cell with higher resistance compared to the rest of the cells in the stack and operating under a negative voltage will be prone to degradation. Thus, the main objective of current work is to advance a fundamental mechanism of degradation of SOEC, which explains the observed delamination of the oxygen electrode.

\subsection{Introduction}

\subsubsection{Global Non-equilibrium, Local Equilibrium}

The proposed degradation model is based on the very fundamentals of non-equilibrium thermodynamics and transport theory. The approach used in this model is similar to the one used previously by the author for studies on transport through predominantly ionic conductors and degradation of solid oxide fuel cell stacks. ${ }^{68,69}$ The approach is based on the incorporation of the local equilibrium criterion into the transport equations. ${ }^{69,70,71}$ Most of the work on transport is based on linear, nonequilibrium thermodynamics. All reported work on transport through solid electrolytes and mixed ionic electronic conductors is based on either explicit or implicit assumption of local equilibrium, even though the implications of this very important assumption are rarely addressed. Recent non-equilibrium molecular dynamics simulations in several systems have shown that local equilibrium applies to a vast majority of the systems that are in global thermodynamic non-equilibrium. ${ }^{72,73,74}$ An important consequence of the existence of local equilibrium in solid electrolytes is that electronic conduction cannot be assumed to be identically zero, even in a predominantly ionic conductor. ${ }^{69,70}$ This is a subtle but very important point. Yet in many studies, contradictory assumptions of the simultaneous existence of local equilibrium (often tacitly made or even apparently unknowingly made) and purely ionic conduction (no electronic transport) are made. ${ }^{75}$

The basic concept of local equilibrium, extensively discussed in textbooks on irreversible thermodynamics, is that even in systems not in global equilibrium Gibbsian laws of thermodynamics apply to microscopic volumes suitably chosen. This is the criterion of local equilibrium in an otherwise global non-equilibrium. In such cases, all extensive thermodynamic functions are replaced by their local densities. The implications of local equilibrium in predominantly oxygen ion conductors in a globally non-equilibrium state, which forms the basis for the proposed electrode delamination model, is discussed below. The general conclusion of non-equilibrium thermodynamics is that local equilibrium is almost always valid, even in cases involving nonlinear, non-equilibrium thermodynamics. In fact, the few (possible) cases in which local equilibrium may be violated are very rare and not encountered in cases involving transport of matter by diffusion. All transport studies assume local equilibrium. 


\subsection{Analysis of Currents through a Cell}

\subsubsection{Ionic and Electronic Currents through the Cell and the Measured Current}

In what follows, the terminology used will be generic: steam- $\mathrm{H}_{2}$ electrode, oxygen electrode, and electrolyte. In the fuel-cell mode, the oxygen electrode is the cathode and the steam- $\mathrm{H}_{2}$ electrode is the anode. In the electrolyzer mode, however, the oxygen electrode is the anode and the steam- $\mathrm{H}_{2}$ electrode is the cathode. Since the focus of this manuscript is on the electrolyzer, the oxygen electrode is nominally the anode and the steam- $\mathrm{H}_{2}$ electrode is nominally the cathode.

Figure 8 shows a schematic of a solid oxide electrolyzer cell. Figure 9 is an equivalent circuit for the electrolyzer cell in a true steady state with an externally applied voltage source, $E_{A}{ }^{a}{ }^{a}$ In Figure $9, r_{i}^{c}$ and $r_{i}^{a}$ are respectively the area-specific ion charge transfer resistances at the steam- $\mathrm{H}_{2}$ electrode/electrolyte interface and the oxygen electrode/electrolyte interface. As-defined, these only refer to the physically sharp electrolyte/electrode interfaces and do not include the porous electrode contributions to the overall electrode reactions. ${ }^{70}$ This is because one must then include simultaneous and parallel transport through both the solid (ions and electrons/holes) and the porous (gas) regions of the electrodes. The resistances, $r_{i}^{c}$ and $r_{i}^{a}$ are effectively polarization resistances (excluding the porous electrodes) and may be described using Butler-Volmer type of phenomenological models. The $r_{e}^{c}$ and $r_{e}^{a}$ are area-specific

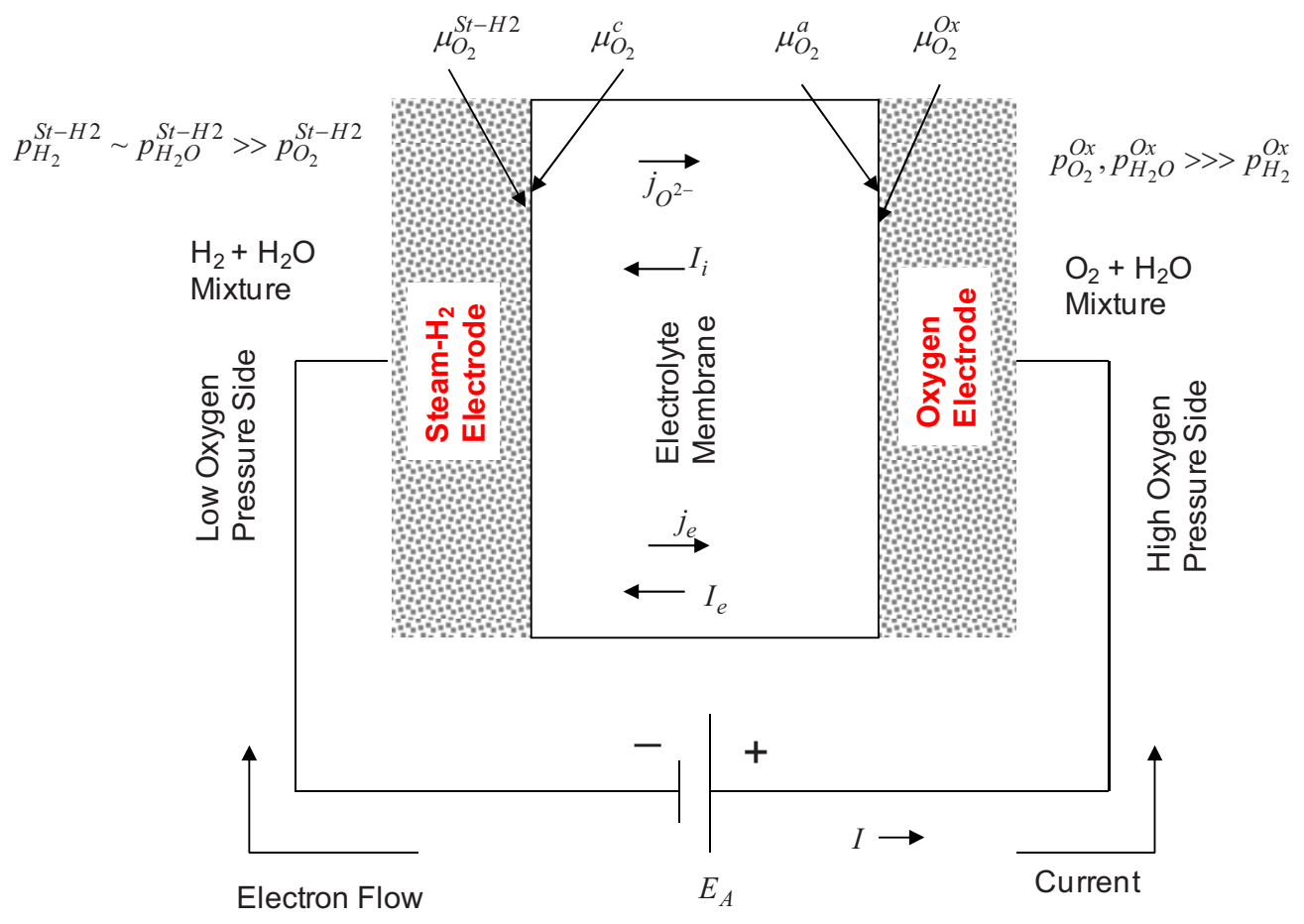

Figure 8. A schematic of a solid oxide electrolyzer cell, when operated in the electrolyzer mode.

a. It is assumed here that the internal resistance of the external source with voltage $E_{A}$ is negligible. It can be easily included in the analysis. 
resistances for direct electron transfer across the steam- $\mathrm{H}_{2}$ electrode/electrolyte interface and oxygen electrode/electrolyte interface, respectively. In the semiconductor terminology, the $r_{e}^{c}$ and $r_{e}^{a}$ are areaspecific contact resistances (non-ohmic contact). Finally, $r_{i}^{e l}$ and $r_{e}^{e l}$ are respectively the ionic and electronic area-specific resistances of the electrolyte, given in terms of the respective conductivities and the electrolyte thickness. Note that $r_{e}^{c}, r_{e}^{a}$, and $r_{e}^{e l}$ may be very large but are not mathematically infinite.

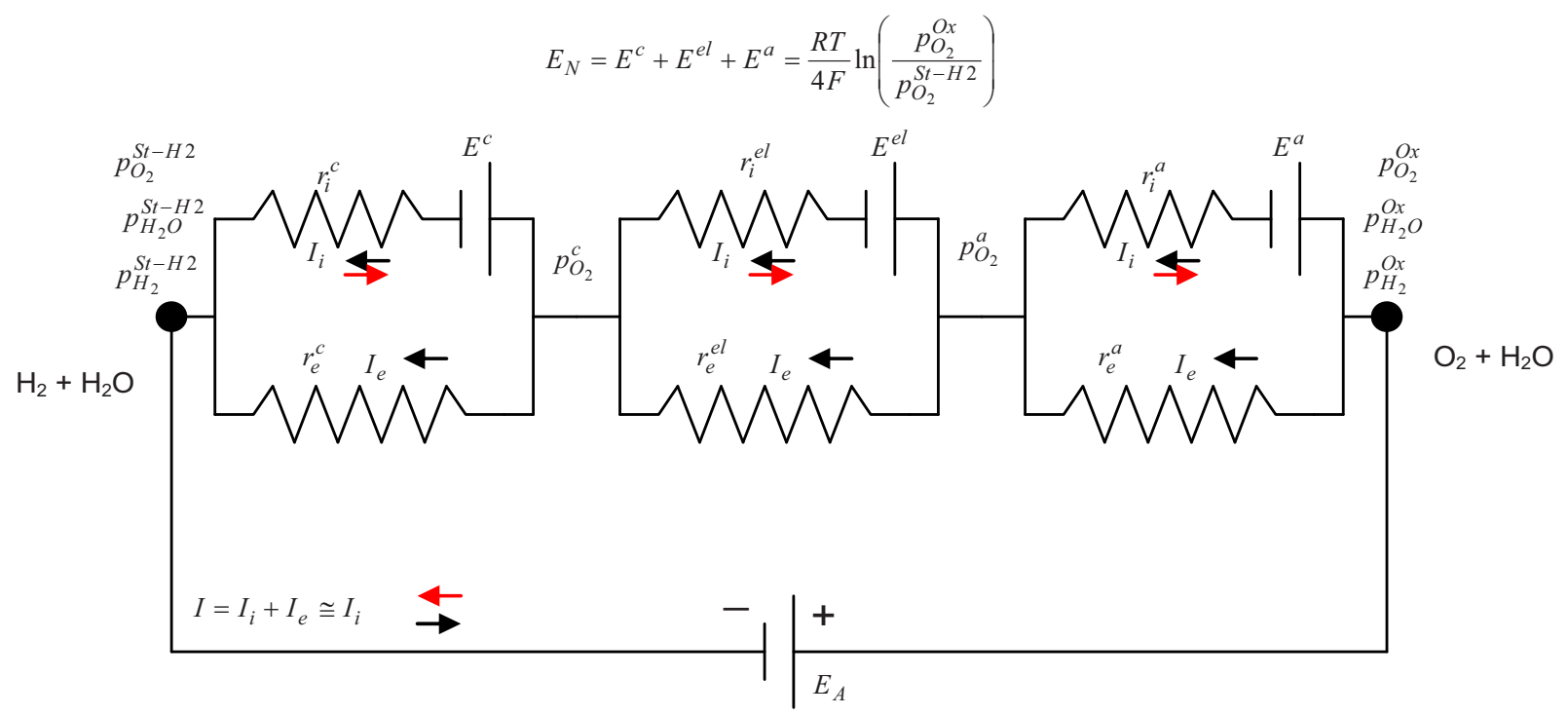

Figure 9. An equivalent circuit for the cell in a "true" steady state.

Figure 10 is an equivalent circuit for the electrolyzer cell in an apparent steady state. The apparent steady state is characterized by a nearly constant voltage across the cell and a nearly constant externally measured current. Since $E_{A}$ is fixed, the voltage across the cell is fixed. However, the electronic current through the electrolyte, which is much smaller than the ionic current, may vary with position and time.

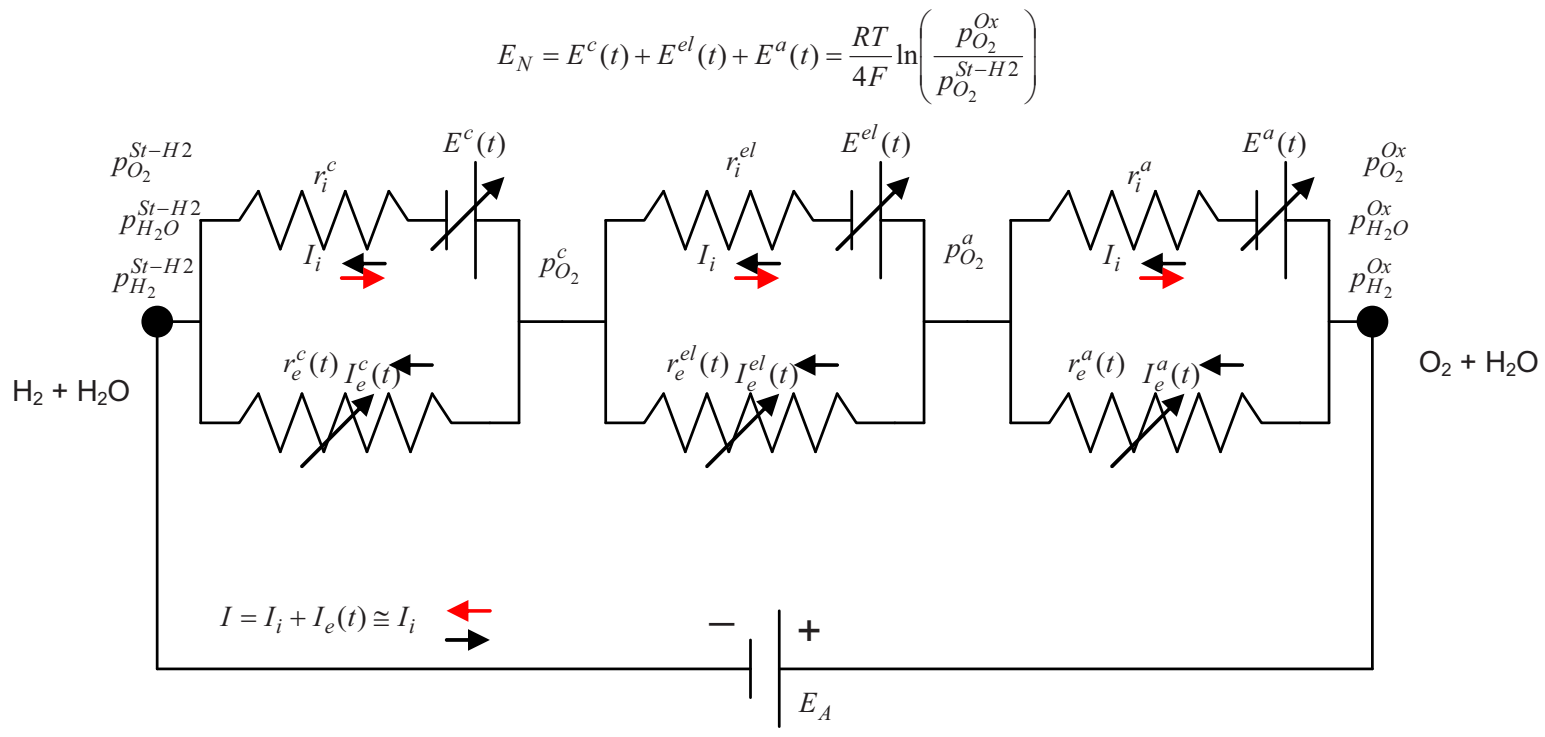

Figure 10. An equivalent circuit for the cell in an "apparent" steady state. 
This effectively reflects as time-dependent individual internal Nernst potentials (with their sum still being a constant, $\left.E_{N}\right)$ and also time-dependent local electric potential, $\varphi(\vec{r}, t)$ (with $\varphi^{O x}-\varphi^{\text {Steam-H2 }}$ fixed and equal to the applied voltage $E_{A}$ ). The following discussion is restricted to the true steady state depicted in Figure 10.

The applied voltage $E_{A}$ is in the opposite direction to the Nernst voltage $E_{N}$ created by differing oxygen partial pressures at the two electrodes; that is, the positive of the cell is connected to the positive of the external source and the negative of the cell is connected to the negative of the external source. When $E_{A}<E_{N}$, the cell does work on the external source (charging the externally connected battery). This is the fuel-cell mode. When $E_{A}>E_{N}$, the external source does work on the cell. This is the electrolyzer mode. When the cell is operated in the fuel-cell mode, the ionic and the electronic currents through the cell are in opposite directions. When the cell is operated in the electrolyzer mode, the ionic and the electronic currents through the cell are in the same direction. In predominantly oxygen ion conductors, the electronic current is small in magnitude, yet it has a significant effect on chemical potentials.

The Nernst voltage generated by differing oxygen partial pressures at the two electrodes is given by

$$
E_{N}=\frac{R T}{4 F} \ln \left(\frac{p_{O_{2}}^{O x}}{p_{O_{2}}^{S t-H 2}}\right)
$$

\subsubsection{Delamination resulting from Development of High Oxygen Pressure in the Electrolyte near the Oxygen Electrode}

$\operatorname{Virkar}^{23}$ determined that delamination may readily occur at the oxygen electrode/electrolyte interface at an applied voltage beyond a certain value. At a typical temperature of electrolysis of $800^{\circ} \mathrm{C}$, for values of

$$
\left\{\frac{E_{A} r_{e}^{a}}{R_{e}}-\frac{\left(E_{A}-E_{N}\right) r_{i}^{a}}{R_{i}}\right\}<-0.106 \mathrm{~V}
$$

(corresponding to $p_{\mathrm{O}_{2}}^{a}=100 \mathrm{~atm}$.) delamination may occur along the oxygen electrode/electrolyte interface. Actually, high pressure develops in the electrolyte just near the oxygen electrode.

Note that if $\left\{\frac{E_{A} r_{e}^{a}}{R_{e}}-\frac{\left(E_{A}-E_{N}\right) r_{i}^{a}}{R_{i}}\right\}$ never becomes negative, delamination will not occur because then the pressure just inside the electrolyte will be lower than $p_{O_{2}}^{O x}$. This refers to the values of applied voltage greater than the Nernst voltage; that is $E_{A}>E_{N}$. The preceding discussion thus shows that if

$$
\left\{\frac{E_{A} r_{e}^{a}}{R_{e}}-\frac{\left(E_{A}-E_{N}\right) r_{i}^{a}}{R_{i}}\right\} \geq 0
$$


or if

$$
\frac{\left(\frac{r_{e}^{a}}{R_{e}}\right)}{\left(\frac{r_{i}^{a}}{R_{i}}\right)}=\frac{r_{e}^{a} R_{i}}{r_{i}^{a} R_{e}}=\frac{\left(\frac{r_{e}^{a}}{r_{e}^{c}+r_{e}^{e l}+r_{e}^{a}}\right)}{\left(\frac{r_{i}^{a}}{r_{i}^{c}+r_{i}^{e l}+r_{i}^{a}}\right)} \geq \frac{E_{A}-E_{N}}{E_{A}}
$$

or if

$$
E_{A}<\frac{E_{N}}{\left(1-\frac{r_{e}^{a} R_{i}}{r_{i}^{a} R_{e}}\right)}
$$

delamination will not occur, which means that a relatively high value of $r_{e}^{a}$ and a relatively low value of $R_{e}$ are preferred. Note that $\frac{r_{e}^{a}}{R_{e}}<1$ and $\frac{r_{i}^{a}}{R_{i}}<1$.

How best to achieve this? The following discussion is presented assuming the electrolyte is YSZ. It is well known that YSZ has a very low electronic conductivity at $800^{\circ} \mathrm{C}$; in fact, it is virtually immeasurable. Thus, $R_{e}$ is very high and it must be lowered to prevent high pressure buildup. If the YSZ electrolyte is doped with ceria (or some other oxide capable of creating some electronic conduction in YSZ) it will lower the $r_{e}^{e l}$ by introducing electronic conduction in YSZ, and this should lower $R_{e}$. If a small amount of $\mathrm{CeO}_{2}$ is added to YSZ, it virtually does not change its oxygen ion concentration. However, a small amount of $\mathrm{CeO}_{2}$ will change electron concentration orders of magnitude (but still in the dilute solution limit) and thus will increase the local Fermi level, $\mu_{e}(\vec{r})$. Thus the role of $\mathrm{CeO}_{2}$ as an additive to the electrolyte is to lower internal pressure, thus lowering the propensity for oxygen electrode delamination.

If a thin (may be a micron or even a fraction of a micron) layer of YSZ (or some other oxygen ion conductor with a very low electronic conductivity) is now deposited on the electrolyte (on the oxygen electrode side), it will drastically increase $r_{e}^{a}$. In this manner, it may be possible to achieve $\frac{r_{e}^{a}}{R_{e}}=\frac{r_{e}^{a}}{r_{e}^{a}+r_{e}^{e l}+r_{e}^{c}}$ close to one if $r_{e}^{a}>>r_{e}^{e l}, r_{e}^{c}$. It is also preferred that $\frac{r_{i}^{a}}{R_{i}}$ be as small as possible.

That is, the charge transfer resistance or the polarization resistance at the oxygen electrode/electrolyte interface should be as small as possible, while the electronic resistance at the oxygen electrode/electrolyte interface should be as large as possible. 
Finally, it may also be desirable to have a somewhat higher electrolyte resistance, $r_{i}^{e l}$. This result suggests that making too thin a YSZ electrolyte may actually slightly increase propensity to electrode delamination. Naturally, the electrolyte cannot be too thick because the performance will be compromised and may also increase the tendency for electrode delamination related to operating conditions as discussed later. Thus, the main focus should be on lowering the charge transfer (polarization) resistance at the perovskite anode (oxygen electrode)/electrolyte interface, and increasing the electronic resistance at the same interface. Materials such as lanthanum strontium cobalt iron oxide LSCF developed for SOFC oxygen electrode (cathode) may be ideal for the electrolyzer oxygen electrode (anode). However, one needs to make sure that the polarization resistance is low for the $\mathrm{O}^{2-} \rightarrow{ }^{1} /{ }_{2} \mathrm{O}_{2}+2 \mathrm{e}$ ' (oxidation) reaction, which is opposite to the SOFC cathode reaction (opposite to the oxygen reduction reaction). Note that it is not at all obvious that the polarization resistance would be the same in both directions (oxidation versus reduction) and may need to be separately determined.

\subsubsection{Example Calculations}

As the electronic resistance of the electrolyte, $r_{e}^{e l}$, is varied between zero and $20 \Omega \mathrm{cm}^{2}$, the cell electronic resistance varies between $1.5 \Omega \mathrm{cm}^{2}$ and $21.5 \Omega \mathrm{cm}^{2}$, the corresponding $\frac{r_{e}^{a} R_{i}}{r_{i}^{a} R_{e}}$ varies between 1.3 and 0.091 (the corresponding measured cell area-specific resistance from voltage versus current curves varies between $\sim 0.31 \Omega \mathrm{cm}^{2}$ and $\sim 0.383 \Omega \mathrm{cm}^{2}$ ), and the corresponding $p_{\mathrm{O}_{2}}^{a}$ varies between $\sim 8.45$ $\times 10^{-17}$ atm. (for $\frac{r_{e}^{a} R_{i}}{r_{i}^{a} R_{e}}=1.3>\frac{E_{A}-E_{N}}{E_{A}}=0.2903$ ) and $\sim 2.3 \times 10^{4}$ atm. (for $\left.\frac{r_{e}^{a} R_{i}}{r_{i}^{a} R_{e}}=0.091<\frac{E_{A}-E_{N}}{E_{A}}=0.2903\right)$.

This result, which shows that changes in electronic conduction characteristics of the cell, which make modest changes in the overall cell resistance as would be measured from voltage versus current density plots or impedance spectra $\left(\sim 0.383 \Omega \mathrm{cm}^{2}\right.$ to $\left.\sim 0.31 \Omega \mathrm{cm}^{2}\right)$, can change the pressure generated in the electrolyte just near the oxygen electrode/electrolyte interface by several orders of magnitude. The conclusion is that under such conditions (high electronic resistance of the electrolyte), delamination along the oxygen electrode/electrolyte interface is imminent and cannot be avoided. In fact, it may be reasonably be expected that delamination will occur above some value of internal pressure $\left(p_{\mathrm{O}_{2}}^{a}\right)$ that is much lower than this value, perhaps say $100 \mathrm{~atm}$. Details of the nature of the interface, defects present, electrode microstructure, and general mechanical properties will determine the pressure above which delamination will occur. ${ }^{b}$ The key conclusion is that no matter what the mechanical properties are, high enough pressures will most certainly be developed to cause oxygen electrode delamination at values of electronic resistance sufficiently high. Indeed, experimental results in several studies have shown that delamination often occurs along the oxygen electrode/electrolyte interface in the electrolyzer mode. ${ }^{1}$ This result suggests that the intuitively obvious approach of trying to develop an electrolyte material with the highest possible ionic transference number may in fact be counterproductive from the standpoint of stability. Thus, electrode delamination is highly likely at $800^{\circ} \mathrm{C}$ with $\mathrm{YSZ}$ as the electrolyte for the

b. Some defects are almost always present. Note, however, degradation by delamination is expected even if no defects are present, since the cohesive strength of most materials is about $10 \%$ of the Young's elastic modulus (which is typically $\sim 10^{5} \mathrm{~atm}$ ). Thus, internally generated pressures on the order of $10^{4} \mathrm{~atm}$. (or greater) will cause cracking (delamination), regardless of the presence of any defects (which are always present). 
operating parameters selected in these calculations. Thus, a small amount of electronic conduction through the electrolyte is actually beneficial.

One should be able to achieve a reasonably high ionic transference number for the membrane while also ensuring stability against delamination and cell degradation. It is a straightforward matter to recognize that significant optimization of parameters is possible, which will not only allow the attainment of a high overall ionic transport number (high electrolysis efficiency), but will also ensure that high internal pressures are not developed under normal electrolyzer operating conditions, thus preventing electrode delamination/degradation. If all relevant parameters are known (which can be experimentally measured), membranes can be designed that will not degrade.

The preceding discussion is given in terms of the required cell transport parameter, namely $\frac{r_{e}^{a} R_{i}}{r_{i}^{a} R_{e}}$, which must be greater than a given operating conditions parameter, namely $\frac{E_{A}-E_{N}}{E_{A}}$, so that oxygen electrode delamination does not occur. This means that if a given set of operating conditions are selected, namely $E_{N}$ and $E_{A}$, then those cells (materials and microstructures, for example) for which $\frac{r_{e}^{a} R_{i}}{r_{i}^{a} R_{e}}$ satisfies the required criteria will not degrade, while those that do not satisfy the required criteria will likely degrade. Alternatively, for a given set of cells, there will be a critical value of $\frac{E_{A}-E_{N}}{E_{A}}$ describing the operating conditions, which should not be exceeded. That is, for a given cell, one must have $\frac{E_{A}-E_{N}}{E_{A}} \leq \frac{r_{e}^{a} R_{i}}{r_{i}^{a} R_{e}}$ or $E_{A}<\frac{E_{N}}{\left(1-\frac{r_{e}^{a} R_{i}}{r_{i}^{a} R_{e}}\right)}$ to prevent oxygen electrode delamination. Since $R_{i}=r_{i}^{c}+r_{i}^{e l}+r_{i}^{a}$ and $R_{e}=r_{e}^{c}+r_{e}^{e l}+r_{e}^{a}$, multiple options exist to select the various transport parameters such that the delamination of oxygen electrode may be prevented.

An important consequence of non-equilibrium thermodynamics is the occurrence of abrupt changes in potentials (chemical and/or electrical) across interfaces. ${ }^{69,70,73,76}$ If the interface is modeled as a thin transition region - perhaps a few nanometers or even a fraction of a nanometer-all changes in the transition region are naturally continuous. However, on a microscopic scale (or even at a submicroscopic scale), smooth changes occur in regions adjacent to the interfaces and sharp changes occur through the interfaces, as has also been shown by non-equilibrium molecular dynamics simulations. ${ }^{73,76}$ Thus, the existence of a very high $\mu_{O_{2}}(\vec{r})$, which is $\mu_{O_{2}}^{a}$ (or $p_{O_{2}}(\vec{r})$, which is $p_{O_{2}}^{a}$ ) in the electrolyte just near the oxygen electrode; and low $\mu_{O_{2}}(\vec{r}+\delta \vec{r})$, which is $\mu_{O_{2}}^{O x}$ (or $p_{O_{2}}(\vec{r}+\delta \vec{r})$, which is $p_{O_{2}}^{O x} \sim 1 \mathrm{~atm}$.) in the oxygen electrode, just across the electrolyte/electrode interface, is perfectly reasonable. Here, $\delta \vec{r}$ is the interface thickness.

\subsubsection{Development of Low Oxygen Pressure within the Electrolyte just near the Steam- $\mathrm{H}_{2}$ Electrode/Electrolyte Interface}

The other possibility for degradation is local electrolyte decomposition if the $p_{\mathrm{O}_{2}}^{c}$ drops below the decomposition pressure for zirconia. At $1073 \mathrm{~K}$, the standard free energy of formation of $\mathrm{ZrO}_{2}$ is 
$894.6 \mathrm{~kJ} / \mathrm{mol}^{77}$ This corresponds to an equilibrium oxygen partial pressure of $\sim 2.8 \times 10^{-44} \mathrm{~atm}$. Thus, to ensure that decomposition does not occur, $p_{\mathrm{O}_{2}}^{c}$ must be above this value. The expected value of oxygen partial pressure in the hydrogen being formed at the steam $-\mathrm{H}_{2}$ electrode is assumed to be $10^{-20} \mathrm{~atm}$ or $p_{\mathrm{O}_{2}}^{\mathrm{St}-\mathrm{H}^{2}} \sim 10^{-20}$ atm. From Virkar, ${ }^{23}$ note that

$$
\frac{R T}{4 F} \ln \left(\frac{p_{O_{2}}^{S t-H 2}}{p_{O_{2}}^{c}}\right)=-\frac{E_{A} r_{e}^{c}}{R_{e}}+\frac{\left(E_{A}-E_{N}\right) r_{i}^{c}}{R_{i}}
$$

Decomposition will occur at the steam- $\mathrm{H}_{2}$ electrode/electrolyte (YSZ) interface (into the electrolyte), provided the above exceeds $1.25 \mathrm{~V}$ at $800^{\circ} \mathrm{C}$. Thus, to prevent decomposition, we must have

$E_{A}\left(\frac{r_{i}^{c}}{R_{i}}-\frac{r_{e}^{c}}{R_{e}}\right)-\frac{E_{N} r_{i}^{c}}{R_{i}}=\left(E_{A}-E_{N}\right)\left(\frac{r_{i}^{c}}{R_{i}}\right)-E_{A}\left(\frac{r_{e}^{c}}{R_{e}}\right) \leq 1.25 \mathrm{~V}$

Equation 42 shows that $\frac{r_{i}^{c}}{R_{i}}$ should be as small as possible and $\frac{r_{e}^{c}}{R_{e}}$ should be as large as possible.

This means the polarization resistance for the oxygen-reduction reaction (at the steam- $\mathrm{H}_{2}$ electrode, should be as low as possible, and the electronic resistance at the steam- $\mathrm{H}_{2}$ electrode/electrolyte interface should be as large as possible. The relative magnitudes of the voltage values show, however, that in general, delamination at the oxygen electrode is the likely mode of degradation rather than decomposition at the steam- $\mathrm{H}_{2}$ electrode/electrolyte interface. This is in accord with the observations in several studies.

\subsection{Implication of Non-equilibrium Thermodynamic Modeling}

In the previous section, the roles of electrolyte and electrolyte/electrode interface transport properties on the possible delamination of the oxygen electrode were analyzed. The fundamental basis of the model developed is the concept of local thermodynamic equilibrium in systems that are not in global thermodynamic equilibrium. This means chemical potentials of the various species are position-dependent (and may also be time-dependent). The simpler problem is that of the steady state so that no time dependence is involved. Global non-equilibrium means fluxes of various species are nonzero and thus the process is irreversible, leading to entropy generation. Kinetics determines the rate at which entropy is generated. If the relationships between fluxes and forces are linear, then the laws of linear non-equilibrium thermodynamics are applicable. This would mean the applicability of Onsager equations. These are also applicable in transient cases as long as flux-force relations are linear. To date, we have examined the steady-state case, with some limited work on transient cases. Ultimately, there is actually a need to address nonlinear non-equilibrium thermodynamics (with still local equilibrium assumed). In such cases, flux-force relations are not linear and may lead to oscillatory behavior. Experimentally, this could reflect as oscillations in voltage at a fixed current, for example. Investigation of these cases is deemed very important but quite outside the scope of the present work.

Central to the model developed is the application of the local equilibrium concept to predominantly ionic conductors. This leads to one important conclusion: Even in a predominantly ionic conductor, electronic conductivity cannot be assumed to be mathematically zero. It may be very, very small - but not zero. Much of the work on fuel cells, batteries, electrolyzers, etc. is based on only including ionic transport. This assumption, however, at worst is incorrect, and at best is incomplete, because it then does not allow one to define local thermodynamics. 
The application of linear non-equilibrium thermodynamics leads to the following key results:

- In a predominantly ionic conductor, when thermodynamic forces exist, electronic current cannot be mathematically zero. Thus, whenever a system is not in global thermodynamic equilibrium, nonzero fluxes exist, including those of electronic species.

- Relative directions of ionic and electronic currents through the ionic conductor determine the local thermodynamics. When ionic and electronic currents are in opposite directions, the chemical potentials of electrically neutral species within the ionic conductor (membrane) are bounded by values in the adjacent reservoirs (electrodes). However, when the ionic and the electronic currents are in the same direction, the chemical potentials of electrically neutral species within the ionic conductor (membrane) need not be bounded by values in the adjacent reservoirs (electrodes). Under certain conditions, chemical potentials within the membrane exceed those in the reservoirs (electrodes). This can lead to instability of the membrane. These results are direct consequences of the coupling of fluxes in accord with the Onsager transport theory. Also, these results are very general and applicable to all electrochemical systems. The author recently has applied this general approach to degradation of lithium-ion batteries. ${ }^{77}$

\subsubsection{Implications concerning solid oxide fuel cells (SOFC) and solid oxide electrolyzer cells (SOEC)}

The membrane in both SOFCs and SOECs is a predominantly oxygen ion conductor, such as YSZ. However, as stated earlier, the electronic conductivity of YSZ cannot be mathematically zero. Thus, some electronic current must flow through the membrane (YSZ) when an electrical potential gradient exists. In the SOFC mode in a single cell, the ionic and the electronic currents through the electrolyte are in opposite directions. Thus, the chemical potential of $\mathrm{O}_{2}, \mu_{O_{2}}$ is mathematically bounded by the values at the electrodes (fuel electrode and oxygen electrode). For this reason, a SOFC is expected to generally exhibit stable behavior (this conclusion is restricted to a single cell-not necessarily for a series connected stack). In the SOEC mode, however, the ionic and the electronic currents are in the same direction. The $\mu_{\mathrm{O}_{2}}$ in the membrane need no longer be bounded by the values at the fuel and the oxygen electrode. Under certain conditions, it may exceed that in air. The extent to which it can exceed some critical value depends upon various transport properties of the membrane and the operating conditions as discussed in detail in the last year's report ${ }^{5}$ and the published paper. ${ }^{23}$ The relations between transport parameters, current densities, applied voltage, and chemical potentials are linear. However, note that the relation between $\mu_{\mathrm{O}_{2}}$ and $p_{\mathrm{O}_{2}}$ is logarithmic.

$$
\begin{aligned}
& \text { Specifically, } \\
& \mu_{O_{2}}=\mu_{O_{2}}^{o}+R T \ln p_{O_{2}}
\end{aligned}
$$

where $\mu_{\mathrm{O}_{2}}^{o}$ at the temperature is the standard state chemical potential of oxygen, corresponding to $p_{O_{2}}^{o}=1$ atm. Thus,

$p_{O_{2}}=\exp \left(\frac{\mu_{O_{2}}-\mu_{O_{2}}^{o}}{R T}\right)$ 
Also note that the relationship between $\mu_{\mathrm{O}_{2}}$ and voltage is also linear. This means that the operating conditions (as dictated by the applied voltage) can have a direct linear effect on $\mu_{\mathrm{O}_{2}}$ but an exponential effect on pressure, $p_{\mathrm{O}_{2}}$. Mechanical stability or integrity (as against fracture) is linearly dependent on stress (fracture stress), which is linearly dependent on any internal pressure generated, $p_{\mathrm{O}_{2}}$. This is a remarkable result as it shows that mechanical stability is exponentially related to electrical parameters such as voltage. An example of this is given below:

$$
p_{O_{2}}^{c}=p_{O_{2}}^{o} \exp \left[\frac{4 F}{R T}\left\{\left(\varphi^{c}-\varphi^{I}\right)-\frac{\left(E_{A}-E_{N}\right) r_{i}^{c}}{R_{i}}\right\}\right]
$$

where $E_{A}$ is the applied voltage, and $p_{O_{2}}^{c}$ is the pressure of oxygen in the electrolyte just under the oxygen electrode (other parameters in Equation [3] are given in Reference 23). A modest increase in $\mu_{O_{2}}$ can cause a large increase in $p_{\mathrm{O}_{2}}$. It is easy to show that $p_{\mathrm{O}_{2}}$ on the order of several hundred or thousand atmospheres can be realized in small localized regions. The most likely location of this high pressure is in the membrane just under the oxygen electrode. Should this occur, delamination of the oxygen electrode will occur. Indeed, in many experimental studies, oxygen electrode delamination has been observed. $\operatorname{Virkar}^{23,78}$ analyzed this mechanism quantitatively and identified a range of membrane properties that will ensure that high $\mu_{O_{2}}$ (and thus high $p_{O_{2}}$ ) does not develop. If this is achieved, it should be possible to minimize (or possibly prevent) oxygen electrode delamination. The key conclusion is that some amount of electronic current through the membrane is necessary. That is, the traditional approach of attempting to find an ionic conductor with the highest possible ionic conductivity and the lowest possible electronic conductivity is actually not advisable. What is desired is a membrane that has gradation in transport properties; electronic conductivity of the membrane should be a function of position-it should be somewhat high in contact with the fuel electrode but low in contact with the oxygen electrode.

\subsubsection{Prevention/Suppression of Oxygen Electrode Delamination}

Based on the model, the following recommendations are made for the design of degradation-resistant SOEC:

1. The electrolyte must exhibit some level of electronic conductivity. This increases the chemical potential of electrons (Fermi level), which effectively lowers the chemical potential of oxygen and thus lowers the internally generated pressure under the oxygen electrode. Based on this model, YSZ is not the ideal electrolyte for SOEC.

2. The electron transfer resistance at the oxygen electrode should be as high as possible. That is, the interface should allow the transport of oxygen ions but not of electrons. This also enhances the overall faradic efficiency.

3. The most desirable electrolyte is such that its ionic conductivity is about the same through the thickness, however the electronic conductivity is graded.

4. The operating parameters should be selected so that the internal oxygen pressure is kept below some critical value. This depends upon the operating voltage, which should be maintained below some critical value. This is determined by various ionic and electronic transport properties of the electrolyte and the interfaces. 
The prospects of developing viable electrolyzer systems are excellent. This would, however, require the development of cells with carefully tailored transport properties. The fundamental basis for the design of such cells has been established in the present work.

\subsection{Summary of Non-equilibrium Thermodynamic Modeling}

Based on the model for oxygen electrode delamination in SOECs presented in this report, the following are summary statements that describe essential features of the model, its predictions, and implications concerning the design of robust SOECs.

The model shows that degradation/failure of a SOEC will primarily manifest as delamination along the oxygen electrode/electrolyte interface. Oxygen electrode delamination occurs as a result of the formation of high internal oxygen pressure within the electrolyte, just near the oxygen electrode/electrolyte interface.

The higher the electronic conductivity of the electrolyte (cell), the lower the tendency for the formation of high internal pressures. Preliminary calculations show that modest changes in electronic conduction can cause orders of magnitude changes in oxygen pressure, $p_{\mathrm{O}_{2}}^{a}$. Thus, the present analysis shows that a small amount of electronic conduction through the electrolyte is actually preferred from the standpoint of stability. This is a significant result as it suggests that an oxygen ion conductor of the highest possible ionic transport number (negligible electronic transport number) may be more prone to degradation (oxygen electrode delamination) and thus is not the desired material as an electrolyte. In this context, YSZ may not be the ideal material as electrolyte for SOEC, especially at $800^{\circ} \mathrm{C}$ and lower temperatures.

The addition of a small amount of a transition metal oxide or other oxides with cations exhibiting multiple valence states, such as ceria, to the electrolyte should increase its electronic conductivity and decrease the tendency for the delamination of oxygen electrode. It should be possible to suitably tailor electronic transport through the cell to minimize tendency for high pressure buildup and simultaneously ensure a relatively high ionic transport number for the cell and thus a high electrolyzer efficiency. This can be achieved by depositing a very thin layer of a purely oxygen ion conductor with a high electronic resistance on the base electrolyte on the oxygen electrode side.

The overall propensity for oxygen electrode delamination can be described by a parameter given in terms of the various transport parameters, namely, $\frac{r_{e}^{a} R_{i}}{r_{i}^{a} R_{e}}$. The higher the $\frac{r_{e}^{a} R_{i}}{r_{i}^{a} R_{e}}$, the smaller the propensity for oxygen electrode delamination. In terms of the operating parameters, delamination should not occur as long as $\frac{E_{A}-E_{N}}{E_{A}}<\frac{r_{e}^{a} R_{i}}{r_{i}^{a} R_{e}}$. Alternatively, the applied voltage must satisfy the following condition, namely $E_{A}<\frac{E_{N}}{\left(1-\frac{r_{e}^{a} R_{i}}{r_{i}^{a} R_{e}}\right)}$, to prevent oxygen electrode delamination. Experimental methods can be devised in principle to measure the required cell parameters, namely $r_{e}^{a}, r_{i}^{a}, R_{i}$ and $R_{e}$; and identify safe operating regime for SOECs. 


\section{SUMMARY OF DEGRADATION MODELING IN SOEC}

A unique combination of equilibrium thermodynamics, non-equilibrium diffusion modeling, and first-principles, atomic-scale calculations was used to understand the degradation mechanisms in the SOEC devices and provide recommendations on how to inhibit them. In particular, some practical recommendations are being given on how to modify the SOEC multicomponent systems to minimize $\mathrm{Cr}$ transport and to inhibit the oxygen delamination process. These conclusions will help increase the lifetime of SOEC devices for practical applications.

The non-equilibrium model shows that the degradation/failure of SOECs is primarily the result of delamination along the oxygen electrode/electrolyte interface. Oxygen electrode delamination occurs as a result of the formation of high internal oxygen pressure within the electrolyte, just near the oxygen electrode/electrolyte interface.

Preliminary calculations show that a small amount of electronic conduction through the electrolyte improves the SOEC's stability. It suggests that an oxygen ion conductor of the highest possible ionic transport number (negligible electronic transport number) may be more prone to oxygen electrode delamination and is thus not as desirable a material as an electrolyte. In this context, YSZ may not be the ideal material as electrolyte for SOEC, especially at a temperature of $800^{\circ} \mathrm{C}$ or lower.

Both models indicate that the addition of a small amount of a transition metal oxide or other oxides with cations exhibiting multiple valence states, such as ceria, to the electrolyte should increase its electronic conductivity and decrease the tendency for delamination of the oxygen electrode. It should be possible to suitably tailor electronic transport through the cell to minimize the tendency for high pressure buildup and simultaneously ensure a relatively high ionic transport number for the cell and thus a high electrolyzer efficiency. This can be achieved by depositing a very thin layer of a purely oxygen ion conductor with a high electronic resistance on the base electrolyte on the oxygen electrode side. 


\section{REFERENCES}

1. Guan J., Ramamurthi B., Rudd J., Hong J., Riley P., Weng D. and Minh N., 2007, "High Performance Flexible Reversible Solid Oxide Fuel Cell," GE Global Research Center Report DE-FC3604GO14351, May 15, 2007.

2. Carter, D. et al., 2008, "Determining Causes of Degradation in High Temperature Electrolysis Stacks," Presented at the Workshop on Degradation in Solid Oxide Electrolysis Cells and Strategies for its Mitigation, Fuel Cell Seminar \& Exposition, October 27, 2008, Phoenix, AZ.

3. Gazzarri, J. I., 2007, "Impedance Model of a Solid Oxide Fuel Cell for Degradation Diagnosis," Ph.D. Thesis, University of British Columbia, Vancouver, Canada.

4. Sohal, M. S., 2009, Degradation in Solid Oxide Cells during High Temperature Electrolysis, Idaho National Laboratory Report INL INL/EXT-09-15617, May 2009.

5. Sohal, M. S., A. V. Virkar, S. N. Rashkeev, and M. V. Glazoff, 2010, Modeling Degradation in Solid Oxide Electrolysis Cells, Idaho National Laboratory Report INL/EXT-10-19691, September 2010.

6. Li, X., 2007, “Chapter 28: Fuel Cells," Handbook of Energy Efficiency and Renewable Energy, Boca Raton: CRC Press, Taylor \& Francis Group.

7. Doenitz, W., R. Schmidberger, E. Steinheil, and R. Streicher, 1980, "Hydrogen Production by High Temperature Electrolysis of Water Vapour," Int J. Hydrogen Energy, Vol. 5, pp. 55-63.

8. Isenberg, A. O. 1981, "Energy Conversion Via Solid Oxide Electrolyte Electrochemical Cells at High Temperatures," Solid State Ionics, Vol. 3-4, pp. 431-437.

9. Doenitz, W., and R. Schmidberger, 1982, "Concepts and Design for Scaling Up High Temperature Water Vapour Electrolysis," Int J Hydrogen Energy, Vol. 7, pp. 321-330.

10. Doenitz, W., and E. Erdle, 1985,"High-Temperature Electrolysis of Water-Vapour Status of Development and Perspectives for Application," Int J Hydrogen Energy, Vol. 10, pp. 291-295.

11. Quant, K. H., and R. Stretcher, 1986, "Concept and Design of a 3.5 MW Pilot Plant for High Temperature Electrolysis of Water Vapor," Int J Hydrogen Energy, Vol. 11, pp. 309-315.

12. Masklike, N. J., 1986, "High Temperature Electrolysis Cell Performance Characterization," Int J Hydrogen Energy, Vol. 11, pp. 563-570.

13. Iwahara, H., H. Uchida, and I. Yamasaki, 1987, "High-Temperature Steam Electrolysis (HTSE) using SrCeO3-based Proton Conductive Solid Electrolyte," Int J Hydrogen Energy, Vol. 12, pp. 73-77.

14. Doenitz, W., G. Dietrich, and R. Streicher, 1988, "Electrochemical High Temperature Technology for Hydrogen Production or Direct Electricity Generation," Int J Hydrogen Energy, Vol. 13, pp. 283287.

15. Erdle, E., W. Doenitz, R. Schamm, and A. Koch, 1992, "Reversibility and Polarization Behavior of High Temperature Solid Oxide Electrochemical Cells," Int J Hydrogen Energy, Vol. 17, pp. 817-819.

16. Momma, A., T. Kato, Y. Kaga, and S. Nagata, 1997, "Polarization Behavior of High Temperature Solid Oxide Electrolysis Cells (SOEC),” J. Ceram. Soc. Japan, Vol. 105, pp. 369-373.

17. Kreuter, W., and H. Hofmann, 1998, "Electrolysis: The Important Energy Transformer in a World of Sustainable Energy," Int. J. Hydrogen Energy, Vol. 23, pp. 661-666.

18. Jensen, S. H., P. H. Larsen, and M. Mogensen, 2007, "Hydrogen and Synthetic Fuel Production from Renewable Energy Sources," Int J Hydrogen Energy, Vol. 32, pp. 3253-3257. 
19. Herring, J. S., J. E. O’Brien, C. M. Stoots, G. L. Hawkes, J. J. Hartvigsen, and M. Shahnam, 2007, "Progress in High-Temperature Electrolysis for Hydrogen Production using Planar SOFC Technology," Int J Hydrogen Energy, Vol. 32, pp. 440-450.

20. Shin, Y., W. Park, J. Chang, and J. Park, 2007, "Evaluation of the High Temperature Electrolysis of Steam to Produce Hydrogen," Int J Hydrogen Energy, Vol. 32, pp. 1486-1491.

21. O’Brien, J. E., C. M. Stoots, J. S. Herring, K. G. Condie, and G. K. Housley. The High-Temperature Electrolysis Program at the Idaho National Laboratory: Observations on Performance Degradation, OSTI ID: 961915, DE-AC07-99ID-13727, Report No. INL/CON-09-15564.

22. Mawdsley J. R., J. D. Carter, A. J. Kropf, B. Yildiz, and V. A. Maroni, 2009, "Post-Test Evaluation of Oxygen Electrodes from Solid Oxide Electrolysis Stacks," Int J Hydrogen Energy, Vol. 34, pp. 4198-4207.

23. Virkar, A. V., 2010, "Mechanism of Oxygen Electrode Delamination in Solid Oxide Electrolyzer Cells," Int J Hydrogen Energy, Vol. 35, No. 18, pp. 9527-9543.

24. Sharma, V. I., and B. Yildiz, 2010, "Degradation Mechanism in $\mathrm{La}_{0.8} \mathrm{Sr}_{0.2} \mathrm{CoO}_{3}$ as Contact Layer on the Solid Oxide Electrolysis Cell Anode," J Electrochem Soc, Vol. 157, pp. B441-448.

25. Liu Y. L., A. Hagen, R. Barfod, M. Chen, H. J. Wang, F. W. Poulsen, and P. V. Hendriksen, 2009, "Microstructural Studies on Degradation of Interface between LSM-YSZ Cathode and YSZ Electrolyte in SOFCs," Solid State Ionics, Vol. 180, pp. 1298-1304.

26. Sushko, P. V., A. L. Shluger, and C. R. A. Catlow, 2000, "Relative Energies of Surface and Defect States: Ab initio Calculations of the MgO (001) Surface," Surf. Sci., Vol. 450, p. 153.

27. Frisch, M. J., et al., 2009, Gaussian 09 Reference Manual, Revision A-1, Wallingford: Gaussian, Inc.

28. Kresse, G., and J. Hafner, 1993, “Ab initio Molecular Dynamics for Liquid Metals,” Phys. Rev. B, Vol. 48, p. 13115.

29. Kresse, G., and J. Furthmüller, 1996, "Efficient Iterative Schemes for Ab Initio Total-Energy Calculations using a Plane-Wave Basis Set," Phys. Rev. B, Vol. 54, p. 11169.

30. Kresse, G., and J. Hafner, 1997, “Ab Initio Simulation of the Metal/Nonmetal Transition in Expanded Fluid Mercury," Phys. Rev. B, Vol. 55, p. 7539.

31. Payne, M. C., M. P. Teter, D. C. Allan, T. A. Arias, and J. D. Joannopoulos,1992, "Iterative Minimization Techniques for Ab Initio Total-Energy Calculations: Molecular Dynamics and Conjugated Gradients," Rev Mod Phys, Vol. 64, pp. 1045-1097.

32. Perdew, J. P., K. Burke, and M. Ernzerhof, 1996, "Generalized Gradient Approximation made Simple," Phys Rev Lett, Vol. 77, pp. 3865-3868.

33. Blöchl, P. E., 1994, "Projector Augmented-Wave Method," Phys Rev B, Vol. 50, pp. 17953-17979.

34. Chadi, D. J., and M. L. Cohen, 1973, "Special Points in the Brillouin Zone," Phys Rev B, Vol. 8, pp. $5747-5753$.

35. Jonsson, H., G. Mills, and K. W. Jacobsen, 1998, "Nudged Elastic Band Method for Finding Minimum Energy Paths of Transitions," Classical and Quantum Dynamics in Condensed Phase Systems, Singapore: World Scientific.

36. Gibbs, J. W., 1948, The Collected Works, Vol. 1, New Haven: Yale University Press.

37. Hillert, M., 2008, Phase Equilibria, Phase Diagrams, and Phase Transformations: Their Thermodynamic Basis, 2nd edition, Cambridge: Cambridge University Press. 
38. Saunders, N., and A. P. Miodownik, 1998, CALPHAD: Calculation of Phase Diagrams, New York: Pergamon Press.

39. Andersson, J. O., et al., 1987, “A New Method of Describing Lattice Stabilities," CALPHAD 11, pp. 93-98.

40. Liu, Z.-K., 2009, "First-Principles Calculations and CALPHAD Modeling of Thermodynamics," $J$ Phase Equilib Diff, Vol. 30, No. 5, p. 517.

41. Glazoff, M. V., F. Barlat, and H. Weiland, 2004, "Continuum Physics of Phase and Defect Microstructures: Bridging the Gap between Physical Metallurgy and Plasticity of Aluminum Alloys," Int J Plasticity, Vol. 20, No. 3, pp. 363-402.

42. Engstrom, A., J. E. Morral, and J. Agren, 1997, "Computer Simulations of Ni-Cr-Al Diffusion Couples," Acta Mater, Vol. 45, pp. 1189-1199.

43. Sundman, B., B. Jansson, and J.-O. Andersson, 1985, CALPHAD, Vol. 9, p. 153.

44. Borgenstam, A., A. Engstrom, L. Hoglund, and J. Agren, 2000, "DICTRA: A Tool for Simulation of Diffusional Transformations in Alloys," J Phase Equilib, Vol. 21, No. 3, pp. 269-280.

45. Rashkeev, S. N., K. W. Sohlberg, S. Zhuo, and S. T. Pantelides, 2007,“Hydrogen-Induced Initiation of Corrosion in Aluminum," J Phys Chem C, Vol. 111, pp. 7175-7178.

46. Lu, G., and E. Kaxiras, 2005, "Hydrogen Embrittlement of Aluminum: The Crucial Role of Vacancies," Phys Rev Lett, Vol. 94, p. 155501.

47. Chen, M., A. N. Grundy, B. Hallstedt, and L. J. Gauckler, 2006, "Thermodynamic Modeling of the La-Mn-Y-Zr-O System: Computer Coupling of Phase Diagrams and Thermochemistry," CALPHAD, Vol. 30, pp. 489-500.

48. Mitterdorfer, A., and L. J. Gauckler, 1998, " $\mathrm{La}_{2} \mathrm{Zr}_{2} \mathrm{O}_{7}$ Formation and Oxygen Reduction Kinetics the $\mathrm{La}_{0.85} \mathrm{Sr}_{0.15} \mathrm{Mn}_{\mathrm{y}} \mathrm{O}_{3}, \mathrm{O}_{2}$ (g) \& Unknown; YSZ System," Solid State Ionics, Vol. 111, pp. 185-218.

49. Dash, S., D. D. Sood, and R. Prasad, 1996, "Phase Diagram and Thermodynamic Calculations of Alkali and Alkaline Earth Metal Zirconates," J Nucl Mater, Vol. 228, pp. 83-116.

50. ASM International, 2009, Phase Diagram \#203160, Metals Park, OH: ASM.

51. Andrievskaya, E. R., and L. M. Lopato, "Influence of Composition on the T->M Transformation in the Systems $\mathrm{ZrO}_{2}-\mathrm{Ln}_{2} \mathrm{O}_{3}$ (Ln= La, Nd, Sm, Eu)," J Mater Sci, Vol. 30, pp. 2591-2596.

52. Chen, M., B. Hallstedt, and L. J. Gauckler, 2005, "Thermodynamic Modeling of Phase Equilibria in the Mn-Y-Zr-O System,” Solid State Ionics, Vol. 176, pp. 1457-1464.

53. Chen, M., B. Hallstedt, and L. J. Gauckler, 2005, "CALPHAD Modeling of the $\mathrm{La}_{2} \mathrm{O}_{3}-\mathrm{Y}_{2} \mathrm{O}_{3}$ System," CALPHAD, Vol. 29, pp. 103-113.

54. Grundy, A. N., M. Chen, B. Hallstedt, and L. J. Gauckler, 2005, "Assessment of the La-Mn-O System," J Phase Equilib Diff, Vol. 26, pp. 131-51.

55. Jacob, K. T., and Y. Waseda, 1994, "Phase Relations in the Systems SrO- $\mathrm{Y}_{2} \mathrm{O}_{3}-\mathrm{CuO}-\mathrm{O}_{2}$ and CaO$\mathrm{Y}_{2} \mathrm{O}_{3}-\mathrm{CuO}-\mathrm{O}_{2}$ at $1173 \mathrm{~K}$," J Phase Equilib, Vol. 15, pp. 401-405.

56. Rodrýguez-Carvajal, J., M. Hennion, F. Moussa, and A. H. Moudden, "Neutron-diffraction Study of the Jahn-Teller Transition in Stoichiometric $\mathrm{LaMnO}_{3}$," Phys Rev B, Vol. 57, pp. R3190-3193.

57. Mastrikov, Y. A., 2008, First-Principles Calculations of $\mathrm{LaMnO}_{3}$ Surface Reactivity, Doctoral Thesis, Stuttgart: Max-Planck-Institut für Festkörperforschung.

58. Stevens, R., 1986, Zirconia and Zirconia Ceramics, Manchester, UK: Magnesium Elektron Inc. 
59. Teufer, G., 1962, “The Crystal Structure of Tetragonal $\mathrm{ZrO}_{2}$," Acta Crystallogr, Vol. 15, p. 1187.

60. Xia, X., R. Oldman, and R. Catlow, 2009, "Computational Modeling Study of Bulk and Surface of Yttria-Stabilized Cubic Zirconia,” Chem Mater, Vol. 21, pp. 3576-3585.

61. Yashima, M., S. Sasaki, M. Kakihana, Y. Yamaguchi, H. Arashi, M. Yoshimura, 1994,“OxygenInduced Structural Change of the Tetragonal Phase around the Tetragonal-Cubic Phase Boundary in $\mathrm{ZrO}_{2}-\mathrm{YO}_{1.5}$ Solid Solutions," Acta Cryst, Vol. B50, pp. 663-672.

62. Catlow, C. R. A., 1990, "Atomistic Mechanisms of Ionic Transport in Fast-Iron Conductors," J Chem Soc-Faraday Trans, Vol. 86, pp. 1167-1176.

63. Mori, M., T. Abe, H. Itoh, O. Yamamoto, G. Q. Shen, Y. Takeda, and N. Imanishi, 1999, "Reaction Mechanism between Lanthanum Manganite and Yttria-Doped Cubic Zirconia," Solid State Ionics, Vol. 123, pp. 113-119.

64. Kawada, T., N. Sakai, H. Yokokawa, and M. Dokiya, 1992, "Electrical Properties of Transition Metal Doped YSZ,” Solid State Ionics, Vol. 53-56, pp. 418-425.

65. Lee, C. H., and G. M. Choi, 2000, "Electrical Conductivity of $\mathrm{CeO}_{2}$ Doped YSZ," Solid State Ionics, Vol. 135, pp. 653-661.

66. Van Berkel, F. P. F., Y. Zhang-Steenwinkel, G. P. J. Schoemakers, M. M. A. van Tuel, and G. Rietveld, 2009, "Enhanced ASC Performance at $600^{\circ} \mathrm{C}$ by Ceria Barrier Layer Optimization," ECS Transactions, Vol. 25, pp. 2717-26.

67. Chang, H. C., D. S. Tsai, W. H. Chung, Y. S. Huang, and M. V. Le, 2009, “A Ceria Layer as Diffusion Barrier between LAMOX and Lanthanum Strontium Cobalt Ferrite along with the Impedance Analysis," Solid State Ionics, Vol. 180, pp. 412-417.

68. Wang, S., A. Y. Borisevich, S. N. Rashkeev, M. V. Glazoff, K. Sohlberg, S. J. Pennycook, and S. T. Pantelides, 2004, "Dopants Adsorbed as Single Atoms Prevent Degradation of Catalysts," Nature Materials, Vol. 3, pp. 143-146.

69. Virkar, A. V., 2007, “A Model for Solid Oxide Fuel Cell (SOFC) Stack Degradation,” J Power Sources, Vol. 172, pp. 713-724.

70. Virkar, A. V., 2005, "Theoretical analysis of the role of interfaces in transport through oxygen ion and electron conducting membranes," J. Power Sources, Vol. 147, pp. 8-31.

71. Kondepudi, D., and I. Prigogine, 1998, Modern Thermodynamics: From Heat Engines to Dissipative Structures, John Wiley \& Sons, New York.

72. Tennebaum, A., G. Ciccotti, and R. Gallico, 1982, "Stationary Non-Equilibrium States by Molecular Dynamics: Fourier's Law,” Phys. Rev. A, Vol. 25, No. 5, pp. 2778-2787.

73. Rosjorde, A., D. W. Fossmo, D. Bedeaux, S. Kjelstrup, and B. Hafskjold, 2000, "Nonequilibrium Molecular Dynamic Simulations of Steady-State Heat and Mass Transport in Condensation," J. Colloid and Interface Science, Vol. 232, pp. 178-185.

74. Bedeaux, D., S. Kjelstrup, and J. M. Rubi, 2003, "Nonequilibrium Translational Effects in Evaporation and Condensation," J. Chem. Phys., Vol. 119, No. 17, pp. 9163-9170.

75. Armstrong, R. D., and M. Todd, 1995, Solid State Chemistry, Cambridge: Cambridge University Press.

76. Kjelstrup, S., and D. Bedeaux, 2008, "Non-Equilibrium Thermodynamics of Heterogeneous Systems," Series on Advances in Statistical Mechanics, Volume 16, World Scientific, New Jersey. 
77. Barin, I., 1993, Thermochemical Data on Pure Substances: Parts I and II, Weiheim, Germany: VCH Publication.

78. Virkar, A. V., 2011, "A Model for Degradation of Electrochemical Devices Based on Linear NonEquilibrium Thermodynamics and its Application to Lithium Batteries," J. Power Sources, Vol. 196, pp. 5970-5984. 